Argonne Physics Division Informal Report PHY-1972G July 1972

\title{
MASTER
}

\section{THE SPEAKEASY HELP DOCUMENTS}

by

\author{
J. K. Fink
}

Argonne National Laboratory, Argonne, Illinois 60439 


\section{DISCLAIMER}

This report was prepared as an account of work sponsored by an agency of the United States Government. Neither the United States Government nor any agency Thereof, nor any of their employees, makes any warranty, express or implied, or assumes any legal liability or responsibility for the accuracy, completeness, or usefulness of any information, apparatus, product, or process disclosed, or represents that its use would not infringe privately owned rights. Reference herein to any specific commercial product, process, or service by trade name, trademark, manufacturer, or otherwise does not necessarily constitute or imply its endorsement, recommendation, or favoring by the United States Government or any agency thereof. The views and opinions of authors expressed herein do not necessarily state or reflect those of the United States Government or any agency thereof. 


\section{DISCLAIMER}

Portions of this document may be illegible in electronic image products. Images are produced from the best available original document. 
The facilities of Argonne National Laboratory are owned by the United States Government. Under the terms of a contract (W-31-109-Eng-38) between the U. S. Atomic Energy Commission, Argonne Universities Association and The University of Chicago, the University employs the staff and operates the Laboratory in accordance with policies and programs formulated, approved and reviewed by the Association.

\section{MEMBERS OF ARGONNE UNIVERSITIES ASSOCIATION}

The University of Arizona Carnegie-Mellon University Case Western Reserve University The University of Chicago University of Cincinnati Illinois Institute of Technology University of Illinois Indiana University Iowa State University The University of Iowa
Kansas State University The University of Kansas Loyola University Marquette University Michigan State University The University of Michigan University of Minnesota University of Missouri Northwestern University University of Notre Dame.
The Ohio State University

Ohio University

The Pennsylvania State University

Purdue University

Saint Louis University

Southern Illinois University

The University of Texas at Austin Washington University

Wayne State University

The University of Wisconsin

\section{NOTICE}

This report was prepared as an account of work sponsored by the United States Government. Neither the United States nor the United States Atomic Energy Commission, nor any of their employees, nor any of their contractors, subcontractors, or their employees, makes any warranty, express or implied, or assumes any legal liability or responsibility for the accuracy, completeness or usefulness of any information, apparatus, product or process disclosed, or represents that its use would not infringe privately-owned rights. 
Argonne Physics Division Informal Report PHY-1972G July 1972

\section{THE SPEAKEASY HELP DOCUMENTS ${ }^{*}$}

\section{by}

J. K. Fink

Argonne National Laboratory, Argonne, Illinois 60439



A limited number of copies are available for general distribution. Anyone who desires a copy should contact S. Cohen of the Argonne Physics Division; the PHY Informal Reports are not handled through the ANL Technical Publications Department.

\footnotetext{
Work performed under the auspices of the U. S. Atomic Energy
} Commission. 


\section{TABLE OF CONTENTS}

\begin{tabular}{|c|c|c|}
\hline & & Page \\
\hline I. & INTRODUCTION & 1 \\
\hline II. & SPEAKEASY WORDS DEFINED IN THE & 3 \\
\hline & HELP DOCUMENTS & \\
\hline III. & ONE-LINE DEFINITIONS OF T HE & 5 \\
\hline & SPEAKEASY WORDS & \\
\hline IV. & THE SPEAKEASY HELP DOCUMENTS & 11 \\
\hline & APPENDIX & 47 \\
\hline
\end{tabular}




\begin{abstract}
ABST RACT
The HELP documents provide SPEAKEASY users with concise definitions of most of the words available in the current processors. In this report, the documents are given in a variety of formats to enable. one to find specific information quickly. The bulk of this report consists of computer read out of the HELP library via SPEAKEASY.
\end{abstract}




\section{INT RODUCTION}

The user of an interactive language such as TSO SPEAKEASY needs a means of quickly determining the operations available to him and of easily learning to use particular features. To fill this need, a special library named HELP has been created and attached to all SPEAKEASY processors. The HELP library consists of concise documents that describe each word of the SPEAKEASY language. The interactive user may obtain each document by a simple command from his terminal.

The growth capability of SPEAKEASY has created difficulties in formally documenting all new features as rapidly as they are added. Since the HELP documents are available to all SPEAKEASY processors and thus readily accessible to all users, they have become the primary method of documentation of new features.

This report is a preliminary attempt to supply the information available to these documents to the users of SPEAKEASY in an easily read form. The HELP documents have been obtained via SPEAKEASY programs in three formats. The SPEAKEASY program that generates each of the three sections precedes it.

Section II consists of an index to the words described in the HELP documents. Each entry in the HELP library is given a reference name consisting of eight characters. The reference name is usually identical to the name used in the SPEAKEASY processor. If the SPEAKEASY word being described is more than eight characters in length, the reference name consists of the first eight characters.

Short one-line definitions of the w ords in the HELP library are given in Section III. These one-line definitions a re the first line of each HELP document. Each line contains the word being defined with arguments of its SPEAKEASY call followed by a brief definition. 
The last section consists of the HELP documents themselves. Each document begins with the word being described and gives a concise definition of the word, including all possible SPEAKEASY calls. The word being defined and its arguments are capitalized throughout the document so that they may be easily located. Most documents are structured in levels; each paragraph gives greater detail than its predecessor so an interactive user can halt the printing of a document after a paragraph if by then he has sufficient information. "While the documents are brief and do not go into great detail in describing a word, they give sufficient information to enable a user who is unfamiliar with a feature to employ that feature after reading the appropriate HELP document. For the use'r interested in further information, the HELP documents refer to longer more detailed documents if such documentation exists and is readily available.

* This feature has not, as of the date of this report, been implemented. 
II. SPEAKEASY WORDS DEFINED IN THE HELP

\section{DOCUMENTS}

The words listed on the next pages are those

defined in the HELP documents of SPEAKEASY-3E. * The

SPEAKEASY program by which they were obtained is the following.

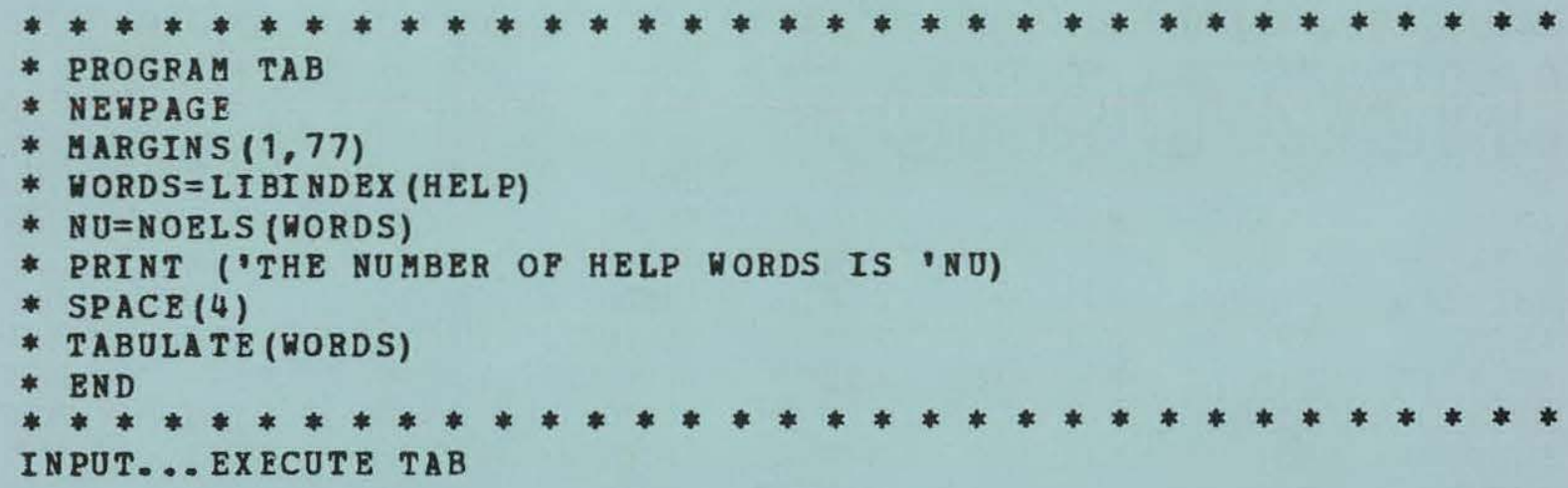

* The versions (i.e. SPEAKEASY $-3 C$ and $3 D$ ) operational at the time of this printing do not employ periods in the definition of logical operators. 
THE NUMBER OF HELP WORDS IS NU $=241$

\begin{tabular}{|c|c|c|c|c|c|c|c|}
\hline WORDS & HORDS & MORDS & HORDS & WORDS & HORDS & WORDS & WORDS \\
\hline ABS & CONJUGAT & ENDAUTOP & INTEGERS & MAT & O MI TCL AS & SETGAUSS & TOTALINT \\
\hline ACCURACY & CONSTRAI & ENDLOOP & INT EGR AL & MATR IX & ONERROR & SETINFIN & TOTINT \\
\hline $\operatorname{Acos}$ & CONTINUE & $E Q$ & INTERP & $\operatorname{MAX}$ & OR & SETJ ACOB & TRACE \\
\hline$\triangle \mathrm{COT}$ & COPY & ER F & I NTER POL & MAXOF & ORDERED & SETLAGUE & TR A NSP \\
\hline$\triangle D D G R \triangle P H$ & $\cos$ & ER PC & INTPART & MAXOFCOL & ORDERER & SETL EGEN & TRANSPOS \\
\hline AFAM & $\mathrm{COSH}$ & EBRORS & INTS & MAXOFROW & PLOTSYMB & SETLIB & UMAT \\
\hline AMT & $\mathrm{COT}$ & EXECUTE & INT2 & MELD & P RI NT & SETNULI & UNITMAT \\
\hline AND & CREATE & EXP & INT4 & MFAM & PRI NTCLA & SETPLOT & UPPERTRI \\
\hline ARRAY & CR EATEME & FIN & I N VER SE & MIIN & PRO D & SIGN & USE \\
\hline ARRAY $2 D$ & CUMPROD & FOR & KEEP & MINOF & PRO DCOLS & SIGNIFIC & USEMEMBE \\
\hline ASIN & CUMSUM & FRAC & KEPT & MINAFCOL & PRODROHS & SIMEQ & VAR IABLE \\
\hline ASYMMAT & DATA & PR ACP ART & LABEL & MINOFROW & P RO GRA M & SIN & VEC \\
\hline ATAN & DEC & FR EE & LE & MOVE & PUNCH & SINH & VECTOR \\
\hline AUTOCORE & DEGRID & GAMMA & L BNGT H & MY DOCS & QUIT & SIZE & VERSIONS \\
\hline AUTOPRIN & DELETE & GE & LIBINDEX & MYHDLP & RAN DOM & SHAT & VFAM \\
\hline AUTOTAB & DERIV & GE IGEN & LIBNAMES & MYLINKS & R AN KED & SOLINDE 2 & VLABEL \\
\hline$A V E R A G E$ & DER IVATI & GOTO & LINKLIB & NA MES & RAN KER & SPACE & VOCABULA \\
\hline$A 1 D$ & DET & GR APH & L INKU L BS & NE & REA D & SPHBES & VSCALE \\
\hline$A 2 D$ & DIAGELS & GRID & LIST & NEU AN N & REA L & S PHB ESN & VSIZE \\
\hline BESSEL & DIAGMAT & GT & LISTHEAD & NEWGRAPH & REALPART & $S Q R T$ & WHERE \\
\hline BESS EL K & DIFFEQ & HELP & LISTMEMB & NEWPAG E & R EA L4 & SUM & WHEREVER \\
\hline CGAMMA & DMAT & HENCEFOR & LOADDATA & NOCOLS & R EA L 8 & SUMCOLS & WHOLE \\
\hline CLEAR & DOCUMENT & HI ERARCH & LOC & NOECHO & RECLASS & SUMROWS & WRITE \\
\hline CLEARDAT & DOHAIN & HI ABEL & LOCMAX & NOELSS & R ET UR N & SUMSQ & ZEROS \\
\hline COLARRAY & DUMP & HSCALE & L OCMI N & NOLI ST & ROOTS & SUMS QCOL & \\
\hline COLMAT & ЕСНо & HS IZ E & LOCS & NOROOTS & ROW ARRAY & SUMSQROW & \\
\hline COLMAX & EDIT & IF & LOG & NOROWS & R OW MAT & SYMBOLS & \\
\hline COLMIN & EIGENSYS & IM A G & L OGGA MMA & NOT & ROW MAX & SYMMAT & \\
\hline COLWIDTH & EIGENVAL & IMA GPART & LOHER TRI & NOZBROS & ROW MI N & TABU LATE & \\
\hline COMPILE & EIGENVEC & INSBRT & LT & NUMBER S & $\mathrm{R} \cup \mathrm{N}$ & TAN & \\
\hline CONJ & END & IN TEG & MARGINS & OBJBCT & SELECT & TIME & \\
\hline
\end{tabular}


III. ONE-LINE DEFINITIONS OF THE SPEAKEASY WORDS

On the following pages are one-line definitions of the words in the HELP documents. The first word of each sentence is the word being defined. The word is written with arguments used in a typical SPEAKEASY call. The SPEAKEASY program which lists the filst line of each HELP document and thus generates these one-line definitions is below.

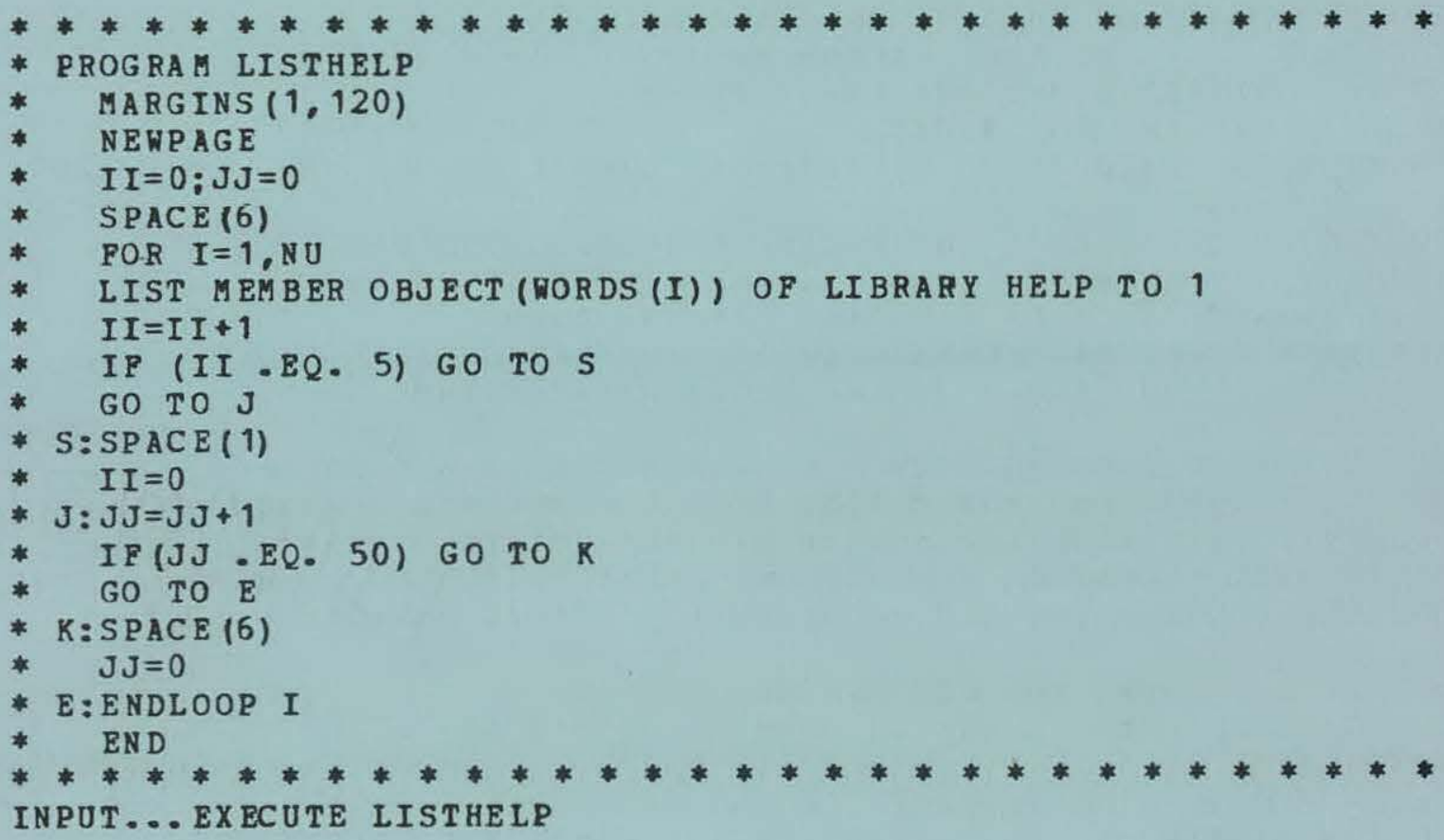


ABS $(X)$ returas the absolute value ofl $X$. ACCORACY (VAL) specifies the smallest number not to be taken as zero. $\Delta \operatorname{Cos}(x)$ defines the arc cosine of $x_{0}$ : $A C O T(X)$ defines the arc cotangent of $x$. ADDGRAPH(I:J) plots I versus $J$ on the previous graph.

APAM $(X)$ defines a member of the array family. AMAT is a synonym for ASYMMAT. -AND. is the logical operator "a nd". ARRAY (N:) defines a $1-d i m e n s i o n a l N^{2}$ component array. ARRAY 2D $(N, M:)$ defines a 2-dimensional $N-b Y-M$ array.

$\operatorname{ASIN}(X)$ defines the arc sine of $X$. ASYMMAT (N: $I, J, \ldots ., K)$ defines an $N-b y-N$ antisymetric matrix. ATAN $(X)$ defines the arc tangent of $X$. AOTOCORE specifies that the data storage space is in LCS. AUTOPRINT $(X, Y, \ldots, Z)$ prints $X, Y, \ldots, Z$ earch $t i m e$ they are redefined.

AUTOTAB specifies uniform tabulations.

AVERAGE (X) returns the average value of the elements of $x$. A $1 D(N:)$ defines a $1-d i m e n s i o n a l$ array. $A 2 D$ is a synonym for ARRAY2D.

BESSEL (NU,X) calculates cylindrical Bessel fn. of the first kind.

BESSELK $(N O, X)$ returns the modified Besse 1 function $K$ of $x$. CGAMMA (X) returns the gama function of $X$ ( $X$ is complex). CLEAR erases the entire active data storage.

CLEARDATA frees all previously defined numerical data. COLARRAY (N:) defines a 2-dim. N-component colum array.

COLMAT (N:) defines an $N-b y-1$ matrix which is a column. COLMAX $(X)$ specifies the column with the maximum element of $X$. COLMIN $(X)$ specifies the column with the minimum element of $x$. COLHIDTH(N) specifies the minimum column width to be used in printing. xCOMPILE returns the processor to the MANUAL mode from EDIT.

CONJ is a synonym for CONJUGATE.

CONJUGATE(X) returas the complex conjugate of $X$.

CONSTRAIN $(A, B, \ldots, C: X)$ constrains $A, B, \ldots, C$ according to condition $X$. CONTINUB causes no operation to be performed.

\%COPY $N, M, K(I)$ copies statements $N$ through $M$ to $K, K+I, K+2 I, \ldots$

$\cos (x)$ returns the cosine of $x$.

$\cos H(x)$ defines the hyperbolic cosine of $x$.

$\operatorname{COT}(X)$ returns the cotangent of $X$.

CREATE MEMBER $X$ ON LIBRARY $Y$ creates a new member from cards.

CREATEMBMBER (XX,YY) creates member XX in library YY.

CUMPROD $(X)$ defines the cumulative product of the elements of $x$. CUMSUM $(X)$ defines the cumulative sua of the elements of $X$. DATA NAME marks the begining of a data file.

DEC (ZA1,ZA2,....ZAN) converts $11, A 2 \ldots . . . A N$ from hex to decimal. DEGRID (XO, XMAX,STEP) gives a grid for DIFFEQ.

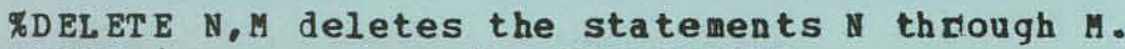
DERIV is a synony for DERIVATIVE.

DERIVATIVE $(F: X)$ finds the derivative of $F$ with respect to $X$. $\operatorname{DET}(X)$ defines the determinant of the atrix $X$. DIAGELS $(x)$ selects the diagonal elenents of $x$. 


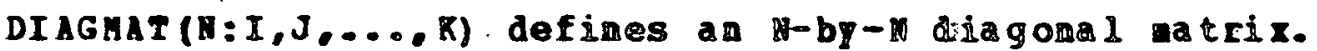

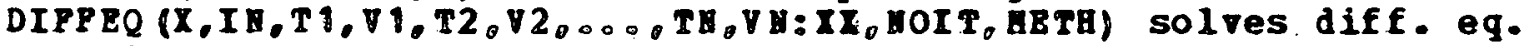
DHAT is a synonym for DIAGRAT.

DOCuGENT is a library of larger documents.

DOMAIN specifies wether real or conplex numbers are being used.

DOMP creates an easily read complete printout of all defined objects. ECHO prints out the input along with: results.

EDIT is a mode available for editing a program.

EIGENSYSTEM (SIMHAT) returns all the eigenvalues of SYHAT.

BIGENVALS $(X)$ gives the eigenvalues of the matrix $X$.

EIGENVECS $(X)$ gives the eigenvectors of the matrix $X$.

END terainates a SPEAKEASY program or datafile.

ENDA UTOPRINT $(X, Y, \ldots, Z)$ tuInS of $\mathrm{Y}$ AUTOPRIAT for objects $X, Y, \ldots, Z$.

ENDLOOP $N$ arks the end of the FOR loop $N$.

-EQ. is the relational operator "equal to".

BRF (X) calculates the error Euncion of $X$.

ERFC (X) calculates the complementary error function of $X$.

ERRORS are ambiguities making it impossible to carry out a statement. EXECUTE NAME executes the stored program, NAME.

EXP $(X)$ defines the exponential function.

FIN is a synonym for ENDLOOP.

POR N=START, STOR, INC designates a loop in a progran.

PRAC is a synonyi for PRACPART.

FRACPART $(X)$ returas the fractional part of $X$.

PREE (N1,N2,.., NE) frees the definitions of the objects $N 1, N 2, \ldots, N N$.

GAMMA $(X)$ defines the gama function of $x$.

.GE. is the relational operator "greater than or equal to".

GEIGEN ( $M, M E T B I C)$ finds the eigenvalues af the matrix $M$.

GOTO $X$ causes execution to be ir ansferred to the statement labeled $x$. GRAPH (I:J) plots the members of I versus the object $J$.

GRID ( $\mathrm{I}, \mathrm{J})$ defines a $1-d i m$. array of 101 points from $I$ to $\mathrm{J}$. -GT. is the relational operator "greater than".

HELP contains a brief description of the SPBAREASY words.

HENCEPORTH $X$ IS $Y$ redefines the yord $X$ to mean $Y$.

HIEBARCHI is the order in wich mathenatical operations are done.

HLABEL='ANY MESSAGE' labels the horizonbal scale of a graph. HSCALE= (LEPT, RIGHT) specifies the horizantal linits of a graph. BSIZE $X$ specifies the horizontal size of the graph.

IF ( $X$ ) $Y$ is a conditional statement for scalar operations.

InAG $(X)$ returns the inaginary part of $X$.

IHAGPABT $(X)$ returns the imaginary part of $X$.

XINSERT $N(I)$ inserts the statements that follow at $N, N+I, N+2 I, \ldots$ INTEG is a synonym for INTEGRAL.

INTEGERS $(I, J, K)$ defines an array with the integers $I, I+K, I+2 R, \ldots . J$. INTEGRAL $(F: X)$ defines the integral of $P$ with a variable upper limit.

IATERP is a synong for INTERPOL.

INTERPOL $(Y, F, X)$ defines the Eunction $P$ at the points $Y$.

IATPABT (X) returas the integer part of $X$.

IHTS is a synonye for IMTBGBRS.

InT $2(X)$ is a function that returns an integer2 auber. 
INT4 $(X)$ is a function that returns an integer4 auber: IUVBRS $B(X)$ defines the inverse of the matrix $X$. KEEP $X X$ AS YY ON $2 Z$ saves the SPEARBASY object $X X$. KEPT ( $Y Y$ ON $Z Z$ ) retrieves the data stored in data set $Y Y$ on $Z Z$. LABEL refers to a statement label.

-LE. is the relational operator "less than or equal to". LENGTH $(X)$ defines a scalar that is the number of elements in $X$. LIBINDEX (Y) defines the nanes of the members of $Y$. LIBNAMES returns the names of the SPEAKEASY libraries. LINKLIB $B X X$ ' adds the LINKOLE library XX to those available.

LINROLES is a library of operations that are PORTRAN subroutines. LIST UEABER X OF LIBRARY $Y$. PROA. I TO J lists lines $I$ through $J$ OP $X$. L.TSTREAD.(N,XX,YY) lists $N$ lines of member $X X$ of library $Y Y$. LISTHEMBER (XX,YY) lists member $X X$ of library $Y Y$. LOADDATA(A, NAME) loads A with values from the data file

LOC is a synonym for LOCS.

LOCMAX $(X)$ specifies the location of the maximu element of $X$. LOCMIN $(X)$. specifies the location of the minimum elenent of $x$. LOCS $(X)$ gives the indices of the nonzero (true) elements of $X$. LOG $(X)$ returns the natural logarithn of $x$.

LOGGAMA $(X)$ defines the natural logarithm of the gamma function of $x$. LORERTRI $(X)$ returns the lover triangular part of $X$. -IT. is the relational operator "less than". MARGINS enables the user to contol the margins of the output. MAT is a synonym for MATRIX.

GATRIX (N, $A:)$ defines an $N-b y-M$ matrix. $\max (X)$ specifies $t$ he value of the maximum element in $X$. Maxor $(A, B, \ldots, C)$ returns the largest element of $A, B, \ldots . . C$. MAXOPCOL $(X)$ returns the largest elenent in each column of $X$. MAXOFRON $(X)$ returns the largest element in each row of $X$.

MELD $(I, J, \ldots, K)$ gives an odometer ordering of elements of $I, J, \ldots, K$. MFAM(X) defines a memer of the matrix/vector family. MIN ( $X)$ specifies the value of the dinimum element in $X$. MINOF $(A, B, \ldots, C)$ returns the smallest element in $A, B, \ldots, C$. MINORCOL $(X)$ returns the smallest element in each column of $X$.

MINOFBOW $(X)$ returns the smallest element in each roy of $X$. XMOVE $N, M, K(I)$ moves statements $N$ through $M$ to $K, K+I, K+2 I, \ldots$ MYDOCS is the library name for private documents.

MYHELP is the library name for private HELP docugents. MYLINKS is the library name for private linkules.

NAMES prints the names of all currently defined SPEAKEASY objects. -NE. is the relational operator "not equal to". NEUMANN $(N U, X)$ returns cylindrical Bessel $E$ ns. of the second kind. NEWGBAPH causes the next graph to be drawn on a ney area of paper. NEHPAGB causes the printer to start a new page.

Nocols $(x)$ defines a scalar which is the number of columns in $x$. NOECHO suppresses the echoing of input data.

MOBLS( $x)$ defines a scalar equal to the number of elenents in $x$. MoLIST indicates that the progras is not to be printed yith the ontput. MOROOTS(F) gives the nunber of roots of the function $P$. 
yonogs (x) defines a scalar equal to the nomer of rows in $x$. .MOT. is the logical operator "not"::

NOzEBos (F) gives the number of zeros of the function $P$. NunBERS refers to numerical data.

OBJECT $(I, J, \ldots ., K)$ generates an inplace nane.

OHITCLASS suppresses the printing of the class.

ONERROR $(X, Y)$ specifies the action to be taken af ter an error.

. OR. is the logical operator "or".

ORDERED $(X)$ gives the elements of $X$ in increasing order.

ORDERER $(X)$ gives $t$ he indices of the ordered elements of $X$.

PLOTSYHB (N, $H$ ) specifies the syabols used and frequency of points. PRINT(A,B,C,...Z) specifies that $A, B, C, \ldots \ldots$ are to be printed.

PRI ATClass prints the class of structured objects.

PROD $(X)$ defines the product of the elements of $x$.

PRODCOLS $(X)$ multiplies the elements in each column of $x$.

PRODROMS $(X)$ multiplies the elements in each roy of $x$.

PROGRA MABE gives a nane to a progran.

PorCH $(F: X)$ punches the object $X$ on cards in the format $F$.

QDIT terainates execution of a SPEAKEASY progran.

RANDOM ( $X$ ) generates random numbers.

RANKED ( $X)$ gives the elements of $X$ in inoreasing order.

BANKER $(X)$ gives the indices of the ordered elements of $X$.

READ $(P: X)$ reads data from cards punched in format $F$ and puts

REAL is a SYMONYa for REALPART.

REALPART $(X)$ returns the real part of $X$.

REAL $4(X)$ is a function that returns a real 4 nuber.

REAL $8(X)$ is a function that returns a real 8 numer.

RECLASS $(A: B, C, \ldots, Z)$ alters the stracture of $B, C, \ldots, Z$ to

RETORN returns execution to the program calling the stored program. ROOTS $(F: X)$ finds the roots of the function $P$.

RORARRAY (N:) defines a 2-din. N-component array that is a row. ROWHAT(N:) defines a 1-by-N matrix which is a row.

RonMaX (X) specifies the row containing the maximum element of $X$. BowaIN $(X)$ specifies the row containing the minimum element of $x$. XRON is equivalent to XCOMPILE followed by the comand EXECUTE.

SELECT (A,B,....C:I) truncates or expands $A, B, \ldots, C$ using the index $I$. SETGAUSS ( $N, X, H, X L O, X H I)$ returns Gauss-Legendre coords. and veights. SETIRPINTY (VAL) specifies an upper limit to the numbers printed. SETJACOBI (N,X,H,A,B,TX,TR) defines Gauss-Jacobi coords. and reights. SETLAGUERRE (N, $X, B, A)$ returns Gauss-Laguerre coords. and veights.

SETLEGENDRE $(N, X, H)$ returns Gauss-Legendre coord. and weights. SETL IB $(X X, Y Y)$ changes the name of library XX to $Y Y$. SETNULI (VAL) specifies a lower limit to numbers printed. SETPLOT $(X, Y, Z)$ specifies BOX, NOBOX: SCALES, NOSCALES: LINES, POINTS. SIGN $(X)$ specifies whether $X$ is positive or negative.

SIGNIFICANCE(N) gives the number of significant figures to be printed. $S I B E Q(A, B)$ solves a set of simultaneous linear equations.

SIN ( $X$ ) returns the sine of $X$.

SInH(x) defines the hyperbolic sine of $x$.

SIZB $=1, X$ specifies space for data and wust be the first card. 
SHAT is a synonge for sYABAT. SOLIUDB2 (X:A,B,C: Y 1,Y1P:IP) solves $I n+A I+B Y=C$ on grid $X$. SPACE(N) skips. N Iines. SPHBES $(L, X)$ returns the spherical Bessel $f$. of the first $k i n d$. SPHBESN $(L, X)$ returns the spherical Bessel fn. of the second $k i n d$.

$S Q R T(X)$ defines the square root of $X$. sou( $X)$. sums the elements of $X$.

suncol $S(X)$. sums the elements in each colven of $x$. . SOHROWS $(X)$ sums the elements in each roy of $X$. SUMSQ $(X)$ sums the squares of the elementis of $x$.

sonsqCols $(X)$ sums the squares of the elements in each column. SOMSQROHS(X) sums the squares of the elements in each rov. SYABOLS designate mathematical operations or special cards. SYMMAT (N:I,J,....K) defines a symet ric $N-b y-N$ natrix. TABULATE (A,B,...,C) prints 1-dia. objects $A, B, \ldots .$, in tabular fora.

TAN (X) returns the tangent of $x$. TIME gives the time from which one starts. TOTALINT $(Z: X)$ defines the defind te integra 1 of $F$ over the array $X$. TOTINT is a synony for TOTALINT.

TRACB $(X)$ gives the trace of the matrix $x$.

TRANSP is a synony for TRANSPOSE.

TRANSPOSE $(X)$ defines the transpose of $X$.

UBAT is a synonym for UNITHAT.

ONITMAT(N) defines an $N-b y-N$ unit matrix.

OPPEBTRI $(X)$ returns the upper triangular part of $X$.

USE MEMBEB X OF LIBRABY Y causes the deck $X$ to be used as input. USEMEMBER(XX,YY) causes input of the meaber $X X$ of library $Y Y$. VARI ABLE (I,J) defines a 1-dim. array of 101 points from $I$ to J. $\checkmark B C$ is a synony for VECTOR.

VECTOR(N:) defines an $N$-conponent vector.

VERSIONS refers to the various versions of the SPEAKEASY processor. VPAM $(X)$ defines a member of the matrix/vector family.

VLABEL = AAY MBSSAGE! labels the vertical scale.

VOCABULARY generates a list of a 11 currently defined words.

VSCALE= (BOTTOH, TOP) specifies the vertical limits of a graph.

VSIZE=I specifies the vertical size of the graph.

WHERE is a synonym for HHEREVBR.

MEEREVER (X) $Y$ is a conditional statement $E$ or array operations. RHOLE $(X)$ returns the integer part of $X$.

URITE $(F: X)$ prints the defined object $X$ in the format $F$.

ZEROS $(F: X)$ finds the zeros of the function $F$. 
IV. THE SPEAKEASY HELP DOCUMENTS

The HELP documents ${ }^{*}$ of the SPEAKEASY $-3 \mathrm{E}$

follow. They give a brief definition of each word in the HELP

library. This listing of the HELP documents has been obtained

by the following program.

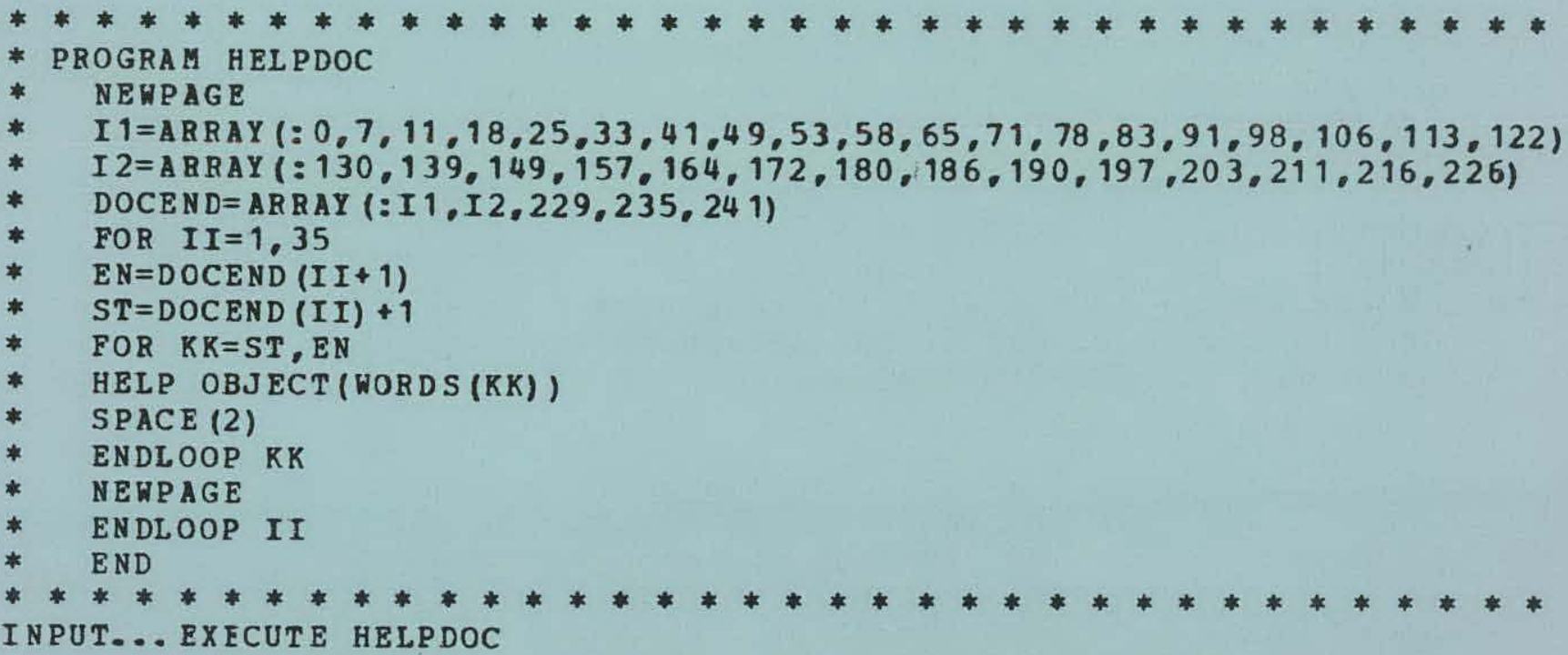

* In the HELP documents the notation $4 \mathrm{E} 60$ is the computer form of the scientific notation $4 \times 10^{60}$. 
ABS ( $x)$ returns the absolute value of $x$. ABS (X) defines an object vith the same structure as $X$ but with elemeats of the result equal to the absolute values of the corresponding elements of $x$.

ACCURACY (VAL) specifies the smallest number not to be taken as zero. Whenever the equality of two numbers is checked during the execution of a program, any number less than a certain number is regarded as zero. The value of this number may be set to be any number VAL by the statement ACCURACY (VAL).

If not specified, the value of VAL is $10 \mathrm{E}-8$.

$\operatorname{Acos}(x)$ defines the arc cosine of $x$. $\operatorname{acos}(X)$ defines an object with the same structure as $x$ with elements that are the arc cosine of the corresponding elements of $x$.

A11 the elements $X i$ of $X$ must be real and satisfy the condition $|x i|<1$ or the condition $|x i|=1$.

$\operatorname{ACOT}(X)$ defines the arc cotangent of $x$. $\operatorname{ACOT}(\mathrm{X})$ defines an object with the same structure as $\mathrm{X}$ with elements that are the arc cotangent of the correspanding elements of $x$.

A11 elements $X i$ of $x$ must be rea 1 .

ADDGRAPH(I:J) plots I versus $J$ on the previous graph. ADDGRAPH has the same meaning as graph exoept a new area of paper is not used. Design statements except the scale statements and the label statements are reexamined prior to adding the graph. Thus the plotting format may be changed for each additional graph.

I is plotted along the vertical axis and $J$ is along the horizontal. All objects should be 1-dimensional and real and have the same number of elements. A 2-dimensional object in $I$ is treated as several 1-dimensional objects each consisting of a row of the original 2-dimensional object. Thus a 2-dimensional object in

I must have as many columins as $J$ has elements.

If no previous graph has been drawn, ADDGRAPH acts as GRAPH.

ADDGRAPH is available only in version GRAPHEZ.

APAM $(X)$ defines a member of the array family. The result has the same structure as $X$ but is an array. If $x$ is a vector or a 1-dimensional array, the result is a 1-dimensional array. If $X$ is a matrix or a 2-dimensional array, the result is a 2-dimensional array.

AHAT is a synonym for ASYMHAT.

AMAT (N:I, J .....K) defines an $N-b y-N$ antisymetric matrix. The element list is used to fill the lower triangular part by rows. The portion above the diagonal is then filled by aking the matrix antisymetric. If any abject in the 1 ist defining the elements is structured, the elements of that structured object are used. 
AND. is the logical operator "and":: The periods aust appear on both sides of the operator.

ARBY (N:) defines a 1-dimensional H-component array. If no further arguments are given, ald the components are set equal to zero.

An alternative form is $A 1 D$.

ARRAY $(: I, J, \ldots, K)$ defines a 1-dim. array and specifies the elements. The elements are preset by the elewent list $I, J, \ldots . . K$. If any argument of the defining element list is structured, the elements of the structured object are used. If a complex element is encountered, then a complez 1-dimensional array is defined.

ARRAY $(N: I, J, \ldots . K)$ defines a $1-d i m . ~ N-c o m p o n e n t$ array. The elements are preset by the element list $I, J, \ldots ., K$. If any argument in the element list it structured, the elements of the structured object are used. If a complex element is encountered, then a complex 1-dimensional array is defined. If all N-components are not specified, the unspecified components are set equal to zero.

ARRAY (N,M:) defines a 2-dimensional N-by-M array. If no further arguments are given, all elements are set equal to zero.

An alternative form is RRAY $2 D$.

ARRAY (N, U:I,J,....K) defines a 2-dim. N-by-M array with preset els. The elements are preset by the element list $I, J, \ldots, K$. If any argument in the element list is structured, then the elements of the structured object are used. If a complex element is encountered, then a two-dimensional $\mathrm{N}^{*}$ by- complex array is defined. All the elements not preset by the element list are set equal to zero.

ARRAY2D(N,M:) defines a 2-dimensional $N$-by-M array. If no further arguments are given, all the elements are set equal to zero.

An alternative form is $\operatorname{RRAY}(N, \mathrm{~N}:)$.

$A$ shortened form is $2 D$.

ARRAY2D(N,M:I, J, ....K) defines a 2-din. $N-$ by-M array. The elements are preset by the element list $I, J, \ldots . K$. If any object of the defining element list is structured, the elements of the structured object are used. If a complex element is encountered, then a 2-dimensional y-by-n complex array is defined. All elements not preset by the element list are set equal to zero.

ASIN $(X)$ defides the arc sine of $X:$ ASIN(E) defines 0 object with the same structure as $x$ with elements that are the arc sine of the correspopding elements of $x$.

All the ele $|x i|<1$ or the condition $f 8 i=1$. 


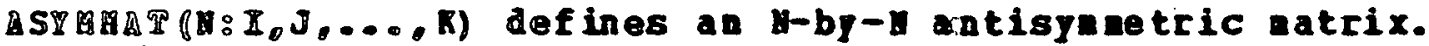
The elevent list is used to fill the lover triangular part by rous. phe portion above the diagonal is then filled by making the erix antisymmetric. If any abject in the 1 ist defining the elements is structured, the eilements of that structured objecs are used.

shortered For is anat.

ATAN (X) defines the aIC tangent of $x$.

arAN $(X)$ defines an object with the sane stiructure as $X$ with elements that are the arc targent of the corresponding elements of $x$.

All elements $\mathrm{Ki}$ of $\mathrm{H}$ must be real.

AOTOCOBE specifies that the data storage space is in LCS. AOTOCORE means that the largest amount of LCS available is to be used for the job.

If $A$ UTOCORE is to be used. it should be the first card in the SPEAKEASY deck.

UTOPBINT $(X, Y, \ldots, Z)$ prints $X, Y, \ldots, Z$ each time. the are redefined. Each object of the name list $X, Y, \ldots \ldots, Z$ is printed whenever it appears on the left of an equation.

AOTOPRINT prints all objects as they are defined or altered. BOTOPRINT is turned off by the statement ENDAOTOPRINT.

AUTOTAB specifies uniform tabulations. The value of $N$ for the coluan width is autonatically set at 10 greater than the numer of significant figures so that the print is uniform for all objects composed of real numbers.

AVERAGE(X) returns the average value of the elenents of $X$. I Is a structured object.

1D 1H: defines a lodimemsiomal array. If mo furtber arguments re giveno alb the components are set equa 1 to zero.

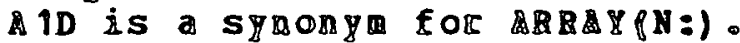


The components are preser by the element list $I, J, \ldots, K$. If any argument in the element list it structured, then the elements of the structured object are used. If a complex element is encountered, then a conplex 1-dinensional array is defined.

A ID (N: $\left.I, J_{0} \ldots, K\right)$ defines a $1-d i m . ~ H-c o z p o n e n t$ array. The components are preset by $t$ he element list $I, J, \ldots . K$. If any argument of the element list itstructured, then the elements of the structured object a re used. If a complex element is encountered, then a complex 1-diainsional array is defined. If all $n$-conponents are not specified, the unspecified components are set equal to zero. 
A2D is a symony for $\triangle B R A B D$.

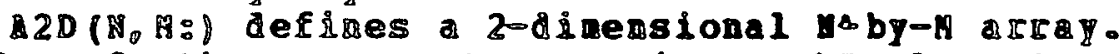

If no further argurents re given, the elements are set equal to reto.

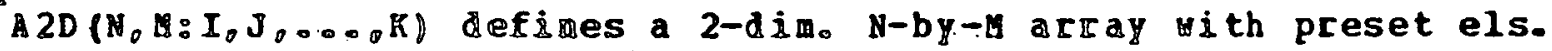
The elements are preser by the elemert list $I_{0} J_{0} \ldots \ldots k$. If any object of the defining element list is structured the elements of the structured object are used:. If a conplex element is encountered. Ehen a 2-dimensional $\mathbb{N}-b y-$ complex array is defined. A 11 elements not preset by the element list are set equal to zero.

BESSEL (NU, $X)$ calculates cylindrical Bessel $f$. of the first kind. $N O$ and $X$ must be rea 1 and nonnegative. There exists a restriction in the SPEAREASY linkule that $(X+N O)<400$.

BESSELK (NO, $X)$ returns the modified Bessel funcrion $K$ of $X$. The resultant is the modified Bessel function $K$ sub $N u$ of $X$. NU and $I$ must be real and nonnegative.: There exists a restriction is the SPRAKEASX linkule that $(X+N 0)<400$.

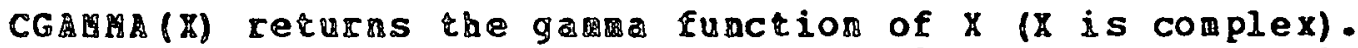
The result has the sane strucerre as 1 with elements equal to the gama Eunction of the corresponding elements of $x$.

If $y$ is real, the gama foraction of $x$ may be obtained from $G$ AMA $(8)$.

CLEA exoses the extire active data storage. Afer the execution of the statement CLEAR there are no user-defined objects, programs, or henceforth definitions.

\section{CLEARDAT Exees ald previously defined numerical data.}

CORAR (R: defines a 2-dim. H-component coluan array. If no further arguents are givero ald N-components are ser equal to zero.

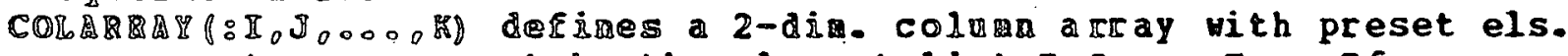
the componers are preser by the element dist $I_{0} J_{0} \ldots ., K$. If 3ny arguent of of that structured object are used. If a conples element is encountered, ther a comex 2-dimensional colum array is defined:

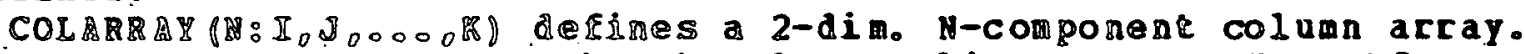
The componeris are preser by the element lisi $\mathbb{I}_{0} \mathbb{J}_{0} \ldots . . K$. If any argument of the element ist is siructured then the elements of that structured object aro used. If a complex element is encompered, eben complex 2-dineasional colum array is defined. IE a d conponents a re not preser by the element list the onspecified comporents are set equal to zero. 
CORAT (N:) defines ar H-by-i atrix which is a colunn. If no Furber arguments are given, all elements are set equal to zero.

CodHat (:I, JoonoR) defines a columa matrix by specifying the els. The elewent list $I_{0} J_{00} \ldots R$ is used to preset the elenents of the column matig. If one of the arguments of the element 1 ist is stractured, the elements of that structured object are used. If one of the elements is complex, the column matrix is complex.

Colnat (N:I, $\left.J_{0} \ldots, K R\right)$ defines an $N-b y * 1$ column matrix yith preset els. The elements are specified by the element list $I, J \ldots . . . K$. If one of the arguments of the 1 ist is structured, the ele me nts of the structured object are used. The column matrix is complex if one of the preset elements is complex. If all $\mathrm{N}$ elements are not preset by the element list, the unspecified elements are set equal to zero.

colmax (X) specifies the colum with the maximum element of $x$.

colmin (x) specifies the column with the minimum element of $x$.

COLHIDTH (W) specifies the minimum column vidth to be used in printing. This statement prevents the colam width of the print routine from being less than $N$ characters. If $N$ is 10 larger than the number of significant figures desired, the print will be uniform for all objects composed of real numbers.

DConpILE returns the processor to the MANOAL node from EDIT. FCOHPILE is used after a program has been edited to return from the EDIT mode to the UANUAL mode.

CONJ is a synonym for conjugatz.

conJ $(X)$ rerurns the complex conjugate of: $x$. CONJ $(y)$ defines an object $y$ ith the same structure as $x$ but with elements equal to the complex zonjugate of the corresponding elerents of

CONJUGRTEPr) returas the complex conjugate of $x$. CONJUGATE(H) defines an object ith the sa me structure as $X$ but with elements equal to the complex conjugate of the corresponding elements ofl $x$.

A shortened form is cons.

ConstaIN $\left(B_{\theta}, \ldots, C: X\right)$ constrains $A, B, \ldots, C$ according to condition $X$. $A_{\theta} B_{0} \ldots . . C$ are 1 -dimensional objects of the same length. $X$ is a logical expression of the same length as the objects to the left of the colon. The objects to the left of the colon are truncated so that they contain only those elements satisfying the logical expression $x$. All 1-dimensional objects must have the same length.

Por an example refer to the SPBARBASY-3 manal. 
COATIMOE causes no operation to be perforned.

The conrinOE statenent is a nonoperational statement to which a label may be attached. Contruob is restricted to the progran mode. It is ignored in the manual mode.

contrue is a restricted vord.

XCOPY $N, M, K(I)$ copies statements through to $K, K+I, K+2 I, \ldots$

XCOPY $N, M, K$ copies statements $n$ through $H$ to $K, K+1, K+2, \ldots$

XCOPY N copies statement $N$ to the last position.

XCOPY $N$, Copies statements $N$ through to the last positions.

XCOPY is a function arailable in the EDIT rode.

$\cos (x)$ returns the cosine of $x$.

$\cos (x)$ defines an object with the same structure as $x$ but

the elements of the result are the cosine of the

corresponding elements of $x$.

Each element $x i$ of $x$ must satisfy the condition

$|X i|<1.815$.

CosH $(x)$ defines the hyperbolic cosine of $x$.

cosH(X) defines an object with the sane structure as $x$ but with elements equal to the hyperbolic cosine of the corresponding elements of $x$.

The elements $X i$ of $X$ must be real and nust satisfy the condition $\mid x$ i $\mid<170$.

$\cot (x)$ returas the cotangent of $x$. cot (X) defines an object with the same structure as $x$ but the elements of the result are the cotangent of the corresponding elements of $X$.

Each element $X i$ of $X$ must satisfy the condition $|X i|<1 . E 15$.

CREATE MEHBER $X$ ON LIBRABY Y creates a new wember from cards. All cards following the card CRBATE MEABER $X$ on LI BRARY $Y$ until the card ENDCREATE is encountered are put in to the member with the na me $X$ in library $Y$.

If the library reference is omitted, it will refer to the library MYROC.

CREATEMEHBER $(X X, Y Y)$ creates member $X X$ in 1 ibrarY $Y Y$. $X X$ is the name of the member that is created.

$P Y$ is the name of the library into which $X X$ is put.

all the cards following the CREATEABABE oard are put into

the member named $X X$ in library $Y Y$ until the card $E N D C R E A T E$

is encountered.

If the library reference is omitted, the member will be put

in the library MYPROCS.

An alternative for is CREATE IREBER XX on LIBRARY YY.

COHPROD(X) defines the cumulative produat of the elements of $x$. CUMPROD $(X)$ defines an object $w$ ith the same structure as $x$ vith elenents that are the cunulatire prodnct of all previous

elements of $x$. Tro-dinensional objects are treated row by row. 
consua ( $x$ ) defines the cunulative sue of the elenents of $x$. cunsua( $X)$ defines an object with the sane structure as $X$ with elements that are the cunulative sun of al: previous elements of $x$. Two-dimensional objects are treated row by rov.

DATA NAUE marks the begining of a data file. NAME is the name given to the data file. The data file is terminated by the single word END. All cards between the card DATA NAME and the card BND contain darta that may be punched in any format. It is read into a specified array by a LOADDATA statement.

DATA is a restricted vord.

DEC (ZA1,ZA2,...ZAN) converts $11, \mathrm{Z} 2, \ldots$ AN from hex to decimal. The hexidecimal numbers A $1, A 2 \ldots .$. AN ust be prefixed by a letter to prevent their conversion to numbers by SPEAKEASY. They may be 7 hexidecinal figures in length. The first character wust be a letter so they are 8-character words.

The result is an array yith elements that are the decimal equivalents of the hex arguments.

DEGRID (XO, XHAX, STEP) gives a grid for DIPFEQ.

$X B A X^{\prime}$ is a 1 -dimensional array of the form $X 1, \times 2, \ldots X N$.

STEP is a 1-dimensional array giving the increments to be added to each Xi: STEP=S1,S2,...,SR.

The result is a 1-dimensional arrag-: The first 48

elements of the result are specially constructed for DIPPEQ. $X(49)=X 0$. The remaining elements are formed by adding $S 1$ to $x 0$ until $\times 1$ is reached: 52 to $\times 1$ until $\times 3$ is reached; etc.

The elements beginning with the 49 th are

$\times 0, \times 0+51, \times 0+251, \ldots . \times 1, \times 1+52, \times 1+252, \ldots, \times 3, \ldots \times \times N$

This is not equivalent to GRID.

TDELETE N,M deletes the statenents through $M$. XDELETE $N$ deletes the statement numbered $N$.

XDELETE is one of the functions available in the BDIT mode.

DERIV is a synonym for DERIVATIVE.

DERIV(P:X) finds the derivative of the $f$ unction $F$ with respect to $X$. $X$ is a 1 -dimensional array and so is the result. Bach element of the result is $d P / d x i$, were $X i$ is the corresponding element of $\mathrm{X}$.

DERIVATIVE (P:X) finds the derivative of $P$ with respect to $X$. $X$ is a 1-dimensional array and so is the result. Each element of the result is $\mathrm{dF} / \mathrm{dXi}$, where $\mathrm{Xi}$ is the corresponding element of $x$.

a shortened form is DERIV.

DET $(x)$ defines the deterninant of the natrix $x$. 
DIAGELS $(X)$ selects the diagonal elenents of $X$. The diagonal elements are given in the order in which they occur in the square matrix $x$. The result is a 1 -dimensional arcay.

DIAGMAT (N: I, J,....K) defines an $N-b y-N$ diagonal matrix. All elements are zero except for those along the diagonal, which are defined by the element list $I, J, \ldots . K$. If any object in the element 1 ist is structured. the elements of that structured object are used.

a shortened form is DMAT.

DIFFEQ (X,IN,T 1,V1,T2,V2,...TN,VN:XX,NOIT, METH) solves diff. eqDIFFEQ solves $N$ coupled differential equations, each of order less than or equal to 4. N must be less than or equal to 10.

The result is an array whose rows contain the solution for each dependent variable evaluated at each point of the grid $x x$.

The kth row of the result contains the solution for the $K t h$ dependent variable evaluated at each point of $X X$.

$X$ is a grid formed by DEGRID.

IN is a 2-dimensional array containing the initial conditions of the dependent variables and their derivatives.

The first column of IN lists the subscript of each variable: the second column lists the order of the derivative; the third column lists the initial value. The ordering of the rows is arbitrary.

$T K$ is a 2-dimensional array referring to the $k$ th equation. TK gives the ordering of the terms.

The first column of TK gives the subscript of the variable in a term; the second column lists the order of the derivative of the variable.

VK is a 2-dimensional array cefering to the Kth equation. The rows of VK consist of the coeficients of the variables in the Kth equation.

The coefficients are evaluated at each point of the grid formed by DEGRID. The first row of $V K$ contains the coefficient of the second term of $T K$; the second row of VK corresponds to the coefficient for the varible of the third row of $T K$; etc.

$X X$ is a grid returned by DIFFEQ.

$X X$ is formed by deleting the first 48 elements of $x$.

NOIT is an optional argument containing the number of corrective iterations. The default value is 2 .

METH is an optional argument giving the degree of the method for each equation. METH is a 1-dimensional array: The default value is the order of the equation + 4 .

For a more detailed explanation and examples, refer to the linkule document.

DMAT is a synonym for DIAGMAT.

DMAT (N:I,J....K) defines an $N-b y-N$ diagonal matrix. All elements are zero except for those along the diagonal. that are defined by the element list $I, J, \ldots, K$. If any object in the element list is structured, the elements af that structured object are used. 
DOCOMENT is a library of larger documents.

The document library contains the documents of the system other than the HELP documents.

DOHAIN specifies whether real or complex numbers are being used. If no domain is specified by the user, it is assumed to be the real domain. The domain may be altered at will by the user by the statements DOMAIN(REAL) or DOMAIN (COMPLEX).

DOMAIN (REAL) specifies that real numbers are to be used. Any calculation leading to an imaginary or complex result is treated as an error.

DOMAIN (COMPLEX) specifies that complex numbers are allowed.

DUMP creates an easily read conplete printout of all defined objects. DuMP enables the user to examine all the information at a given point in a calculation. After the printout, the calculation continues.

DOMP is not available in Tso.

ECHO prints out the input along with results. The first line of output is the user's input. Immediately following are the results or the response to the input.

ECHO is default for batch jobs: NOECHO is default for the interactive operation.

EDIT is a mode available for editing a program. The EDIT mode is autcmatically enterced when one of the words PROGRAM, PROCEDURE, or DATA is encountered. In the EDIT mode statements are assigned successive intejer values starting with 1. The program may be altered by using control functions that are available in the EDIT mode. These are selected by a $\%$ in the first field of the input card. The functions are:

\%IIST

RCOPY

QDELETE

XINSERT

\%MOVE

XCOMPILE

$\mathscr{R} R \mathrm{IN}$

x

For a description of most of these functions, type HELP FUNCTION where PUNCTION is the name of the function desired without $t$ he $\%$. EDIT XX returns the processor to the edit mode with the progran $X X$. $X X$ is the name of a previously def ined program that is to be edited.

The EDIT mode is available on all processors but it is normally not used except for interactive operations. 
EIGENSYSTEM (SYMAAT) returns all the eigenvalues of SYMMAT. The eigenvalues are returned as a vector. If the SPEAKEASY call is $Y=Z I G E N S Y S T E M$ (SYMMAT), then $Y$ is a vector containg the eigenvalues of SYMMAT.

SYMMAT must be a real symmetric matrix.

EIGENSYTEM (SYMMAT: $+M$ ) returns the M largest eigenvalues of SYMMAT. The M eigenvalues are in descending order as the M-components of a vertor.

SYMMAT must be a real symmetric matrix.

EIGENSYSTEM (SYMMAT:-M) returns the loyest eigenvalues of SYMMAT: The $M$ lowest eigenvalues are returned in ascending order as the $M$-components of a vector. SYMMAT must be a real symmetric matrix. EIGENSYSTEM ( $X, E V E C S)$ returns the eigenvalues and eigenvectors of $X$. If the SPEAKEASY call is Y=EIGENSYSTEM ( $X, B V E C S)$, then $Y$ is a vector containg the eigenvalues of $x$. The corresponding eigenvectors are stored row-wise in the $N-b y-N$ matrix, EVECS.

$x$ must be a real symet $r i c$ matrix.

EIGENSYSTEM $(X, V ;+M)$ returns the M largest eigenvalues and eigenvectors. The M largest eigenvalues are returned in descending order as a vector. Their corresponding eigenvectors are returned as the rows of the $M-t y-N$ matrix $V$.

$X$ must be a real symetric matrix.

EIGENSYSTEM ( $X, V:-M)$ returns the $M$ smallest eigenvalues and eigenvectors. The $M$ smallest eigenvalues of $X$ are returned as a vector. $V$ is returned as an $M-b y-N$ matrix containing $M$ eigenvectors corresponding to the M smallest eigenvalues. The eigenvectors are stored row-wise in $v$.

$x$ must be a real symmetric matrix.

EIGENVALS $(X)$ gives the eigenvalues of the matrix $X$. The eigenvalues are listed in order of descending values. $x$ must be a real symetric matrix.

EIGENVECS $(X)$ gives the eigenvectors of the matrix $X$. EIGENVECS $(X)$ defines a matrix whose rows are the eigenvectors of $x$. The resulting $m a t r i x$ is unitary.

END terminates a SPEAKEASY program or datafile. During execution in the program mode, the single word END corresponds to a return. In the manual mode of SPEAREASY, the word END is ignored.

ENDAUTORRINT $(X, Y, \ldots, Z)$ turns of $f$ AUTOPRINT for objects $X, Y, \ldots, Z$.

ENDLOOP $N$ marks the end of the FOR loop $N$. $N$ is the name of the FOR loop that occurs in the corresponding ROR statement.

ENDLOOP is a restricted word.

.EQ. is the relational operator "equal to". The periods must appear on both sides of the operator. 
ERP ( $X$ ) calculates the error function of $x$. The result has the same structure as $x$. with elements equal to the error function of the corresponding elements of $x$.

The elements of $x$ must be real.

ERFC $(X)$ calculates the complementary error function of $x$. The result has the same structure as $X$ with elements that correspond to the complementary error funotions of the corresponding elements of $x$.

The elements of $x$ must be real.

ERRORS are ambiguities making it impossible to carry out a statement. There are two types cf errors in SPEAREASY. They are syntax errors and execution errors. Syntax ercors are those common to all computer languages and are due to misuse of symbols, such as parenthesis imbalance. Such errors are detected before execution of the SPEAKEASY statement. Execution ercors relate to an inconsistency in the use of jefined objects dul dit unique to SPEAKEASY. Errors encountered during compilation of a SPEAKEASY program do not inhibit later execution.

EXECUTE NAME executes the stored program, NAME.

The stored program is executed by this command and continues until a RETURN statement or END. statement is encountered. These statements terminate the execution of the stored program and the statement following the one calling for the execution of the stored program is then executed.

EXECUTE NAME: LABEL executes the program NAME beginning at LABEL. EXECUTE NAME: LABEL causes the stored progran with this name to begin execution at the designated internal: label.

EXECUTE statements may occur in the manual mode or in any stored program. In the manual mode, the Execute statement should occur alone; not as part of a multistatement card.

EXECUTE is a restricted word.

EXP $(X)$ defines the exponential function. EXP(X) defines an object with the same structure as $X$ but with elements equal to the exponential of the corresponding elements of $x$.

Each element $X i$ of $X$ must satisf $y$ the conditions Real $(X i)<170$ and I Imag (Xi) $\mid<5 E 6$.

FIN is a synonym for ENDLOOP. FIN N warks the end of the FOR loop $N$. $N$ is the name of the loop appearing in the corcesponding FOR statement.

FIN is a restricted word. 
FOR N=START, STOP, INC designates a loop in a progra..

$N$ is the nane of a scalar that ay appear in any context in the loop without altering its value. STAR'T, STOP, and

INC are scalar expressions not involving $N$.

START is the initial value of $\mathrm{N}$.

STOP is the final value of $N$.

INC is the increment to be added to $N$ every time the loop is repeated.

If INC is not specified, its value is assumed to be 1.

A FOR loop is terminated by an ENDLOOP statement or by a

PIN statement.

Up to 10 nested FOR loops are allowed. A FOR loop

started within a FOR loop must be terminated within that loop.

since POR loops are neither efficient nor compact in SPEAK EASY. they should be avoided. Logical transfers into and out of

POR loops should be used with extreme caution.

POR is a restricted vord.

PRAC is a synonym for PRACPART.

PRAC $(X)$ returns the fractional part of $X$.

PRAC (X). defines an object with the same structure as $X$ but the elements of the result are the fractional part of the corresponding elements of $X$.

PRACPART $(X)$ returns the fractional part of $X$. FRACPART $(X)$ defines an object with the same structure as $x$ but the elements of the result are the fractional part of the corresponding elements of $x$.

A synonym is FRAC.

PREE (N 1,N2,...,NN) frees the definitions of the objects $N 1, N 2, \ldots, N N$. $N 1, N 2 \ldots . . N N$ are names of defined objects.

GAMMA $(X)$ defines the gamma function of $X$. GAMMA (X) defines an object with the same structure as $x$ but with elements equal to the gama function of the corresponding elements of $x$.

The elements $X i$ of $X$ must be real and must satisfy the condition $10 \mathrm{E}-50<\mathrm{X} i<56$.

.GE. is the relational operator "greater than or equal to". The periods must be on both sides of the operator.

GEIGEN (M, METRIC) finds the eigenvalues of the matrix $M$. $n$ is a real symetric matrix in a nonsorthogonal basis. METRIC is the positive definite matrix of the inner products of the basis states. The eigenvalues of M are returned as the components of a vector.

GEIGEN (M,METRIC,EVECS) finds the eigenvalues and eigenvectors of $M$. $M$ is a real symetric matrix in a nonorthogonal basis. METRIC is the positive definite matrix of the inner products of the basis states. EVECS is a matrix containing the eigenvectors of stored row-vise. The eigenvalues of are returned as the components of a vector. 
Goto $x$ causes execution to be transferred to the statement labeled $X$. The Goto statement is used to alter the sequence of execution of statements. Logical branches are created by combining an IF statement with a GOTO statement.

GoTO is a restricted word.

An equivalent form is Go TO.

GRAPH(I:J) plots the members of I versus the object J.

$I$ is in the vertical direction and $J$ is along the horizontal.

All objects should be 1-dimensional and real and have

the same number of elements. A 2-dimensional object

in I is treated as several 1-dimensional objects,

each consisting of a row of the original 2-dimensional

object. Thus a 2-dimensional object in I must have

as many columns as $J$ has elements.

Each time GRAPH is encountered, a graph is drawn on a

new area of paper. All of the design statements should

accompany GRAPH.

The graphical output from GRAPH in the program mode is plotted by CALCOMP.

GRAPH is available only in version GRAPHEZ.

GRID $(I, J)$ defines a 1-dim. array of 101 points from I to $J$. The increment is chosen by the computer so that the points

are equally spaced. Both points $I$ and $J$ are included.

A synonym is VARIABLES $(I, J)$.

GRID $(I, J, K)$ defines a 1 -dim. array $I, J+K, I+2 K \ldots$ unt il $J$ is reached. The last element is equal to or less than $J$ depending on the increment $K$.

GRID $(I, J, K)$ is not equal to VARIABLE $(I, J ! K)$ unless $K$ is such that $I+N K=J$.

.GT. is the relational operator "greater than". The periods aust be on both sides of the aperator.

HELP contains a brief description of the SPEAKEASY words. An alphabetical. listing of the words in the HELP library ay be obtained by the statements

CATALOG = L IBINDEX (HEIP)

TABULATE (CATALOG)

To obtain the description of a single SPEAKEASY word $X X$, the input statement is

HELP XX

A 11 the words and a description of each way be obtained by

the single statement

USE MEMBER LISTHELP

A 1-line description of a SPEAKEASI word may be obtained

by the statement

LIST MEMBER XX OP LIBRARY HELP TO 1. 
HENCEPORTH $X$ IS $Y$ redefines the word $X$ to mean $Y$. After this statement, anytime the vord $X$ is encountered it will be treated as if it were the word $Y$.

The word 'IS' in the expression is arbitrary. Any word may be used.

Restricted words may not be given synonyims by this method.

HIERARCHY is the order in which mathematical operations are done. The HIERARCHY in SPEAKEASY is:

* raising to a power.

1.* division and multiplication."

+ .- addition and subtraction.

operations of the sawe hierarchy are done left to right.

HLABEL ='ANY MESSAGE' labels the horizontal scale of a graph. The desired label may be anything. Its beginning and end are marked by apostrophes in the specifying statement.

HLABEL is available only in version GRAPHEZ.

HSCALE = (LEFT, RIGH T) specifies the horizontal limits of a graph. LEFT is the left limit and RIGHT is the right limit of the scale. values are labeled at in:h marks. The default limits for the horizontal scale are 0 for the left and 10 for the right.

HSCALE is used for graphical output.

HSIZE $X$ specifies the horizontal size of the graph. $X$ is in inches. The default is 10 inches.

HSIZE is used for graphical output.

IF (X) $-Y$ is a conditional statement for scalar operations. $X$ is a scalar expression. If the numerica 1 value of the expression $X$ is nonzero, then the associated statement $Y$. is carried out; otherwise the statement $Y$ is ignored.

I $P$ is a restricted word.

IMAG $(X)$ returns the imaginary part of $X$. IMAGINARY $(X)$ defines an object with the same structure as $X$ but with elements that are the imaginary part of the corresponding elements of $x$.

A syonym is IMAGPART.

IMAGPART $(X)$ returns the imaginary part of $X$. IMAGPART $(X)$ defines an object with the same structure as $x$ but with elements that are the imaginary part of the corresponding elements of $x$.

A synonym is IMAG. 
XINSERT $N(I)$ inserts the statements that follow at $N, N+I, N+2 I, \ldots$ XINSERT N(I) inserts the statements that follow the words

XINSERT $N(I)$ into the positions $N, N+I, N+2 I \ldots$

XINSERT $N$ inserts the statements that follon at $N, N+1, N+2, \ldots$ XINSERT $N$ inserts the statements that follow the insert comand into locations labeled bY $N, N+1, N+2 \ldots$

* stops the insert.

QINSERT is one of the functions available in the EDIT mode.

INTEG is a synonym for INTEGRAL.

INTEG $(F: X)$ defines the integral of $F$ with a variable upper limit. $X$ is a 1 -dimesional array $(X 1,82, X 3, \ldots, X N)$. The result is a 1-dimensional array the same size as $x$. Each element of the result is the integral from $X_{1}$ to $X i$ where $X i$ is the corresponding element of $x$. The integration is done by the trapezoidal rule.

INTEGERS $(I, J, K)$ defines an array with the integers $I, I+K, I+2 K, \ldots, J$. The array ends when the value $J$ is reached or passed.

INTEGERS $(I, K)$ defines an array ith the integers $I, I+1, I+2, \ldots, K$. If $K$ is less than $I$, INTEGERS $(I, K)$ defines the integer array $I, I-1, I-2, \ldots, R$.

A shortened form is INTS.

INTEGRAL (F: $X$ ) defines the integral of $q$ with a variable upper limit. $X$ is a 1 -dimensional array $(X 1, \times 2, X 3, \ldots, X N)$. The result is a 1-dimensional array the same size as $x$. Each element of the result is the integral from $X 1$ to $X i$ where $X i$ is the corresponding element of $x$. The integration is done by the trapezoidal rule.

A shortened form is INTEG.

INTERP is a synony for INTERPOL.

INTERP $(Y, F, X)$ defines the $f$ unction $F$ at the points $Y$. INTERP.(Y,F, X) is an interpolation method wich is cubic except in the first and last intervals whe re it is quadratic. The result is an array with the values of the function $F$ evaluated at the points $Y$. X $X$ and $Y$ are arrays. If points in $Y$ are outside the range, the interpolation is based on the last 3 foints in the range. $P(X)$ must be given.

INTERPOL $(Y, F, X)$ defines the function $F$ at the points $Y$. INTERPOL $(Y, F, X)$ is an interpolation method which is cubic except in the first and last intervals where it is quadratic. The result is an array with the values of the function $F$ evaluated at the points $Y$. $X$ and $Y$ are arrays. If points in $Y$ are outside the range, the interpolation is based on the last 3 points in the range. $F(X)$ must be given.

A synonym is INTERE.

INTPART $(X)$ returns the integer part of $X$. INTPART(X) defines an object with the same structure as $X$ but the elements of the result are the integer part of the corresponding elenents of $x$.

A synonyw is MHOLE. 
INTS is a synonym for INTEGERS.

INTS $(I, J, K)$ defines an array with the integers $I, I+K, I+2 K, \ldots, J$. The array ends when the value $J$ is reached or passed.

INTS $(I, K)$ defines an array with the integers $I, I+1, I+2, \ldots, K$. If $K$ is less than $I$. INTS $(I, K)$ defines the integer array $\mathrm{I}, \mathrm{I}-1, \mathrm{I}-2, \ldots, \mathrm{K}$.

INT $2(X)$ is a function that returns an in teger2 $n$ umber. $x$ way be real8, real4, or integer 4 . The result is INTEGER2.

INT4(X) is a function that returns an integer4 number. $X$ may be rea14, rea 18, or integer2. The result is integer 4.

INVERSE $(X)$ defines the inverse of the matrix $x$. The result is a matrix.

an alternative statement to obtain the inverse of the matrix $X$ is $1 / X$.

KEEP XX AS YY ON ZZ saves the SPEAKBASY object $X X$. The command means that object $X X$ is to be copied onto data set $Z Z$ and $q$ iven the nade $Y Y$. The object $X X$ is recovered by a KEPT statement.

The data set $Z Z$ is a private data set belonging to the user. It is created by including cards of the form

$/ / Z Z$ DD DS $\mathrm{L}=$ MY NAME, DISP= (NEW, CATLG),

$1 / \quad S P A C E=(T R K,(5,5,2)), D C B=(R E C R M=P B, B L K S I Z E=400, L R E C L=80)$ immediately following the $/ /$ EXEC SPBAKEZ card.

To use the data set, change DISP=(NER,CATLG) to DISP=(OLD,KEEP). To delete the data set when it is no longer needed, use DISP $=$ (OLD, DELETE).

KEPT (YY ON ZZ) retrieves the data stored in data set $Y Y$ on $Z Z$. KEPT (YY ON ZZ) is a normal SPEAKEASY function, which may be imbedded in a statement.

YY is the name assigned to the data in the KEEP statement.

22 is the name of the data set on wich the data are stored.

To use the data set $Z Z$, include a card of the form

$/ / Z Z$ DD DSN=MYNAUE, DI SP= (OLD, KEEP), UNIT $=2.321$

immediately following the // EXEC SPEAKEZ card.

To delete the data set when it is no longer needed, change $D I S P=(O L D, K E E P)$ to DISP=(OLD,DELETE) .

LABEL refers to a statement label. SPEAKEASY statements are labeled by a name of 8 symbols or less, beginning with an alphabetic character. This label is separated from the statement by a colon. The label may be in any column but preceeds the statement.

-LE. is the relaticnal operator "less than or equal to". The periods aust appear on both sides of the operator. 
LENGTH (X) defines a scalar that is the number of elements in $X$. If $X$ is undefined, the result is zero.

A synonym is NOELS.

LIBINDEX (Y) defines the names of the members of $Y$. LIBINDEX ( $Y$ ) defines a literal 1-di mensional array with the names of the members of the library $Y$ as oomponents of the array.

II BNAMES returns the names of the SPEAKEASY 1 ibraries. LIBNAMES defines an array whose elements are the names of the libraries attached to the SPEAKEASY processor.

LINKLIB='XX' adds the LINKOLE library $X X$ to those available. $X X$ is an additional library of linkules that is searched if a designated member is not found in the other libraries.

LINKULES is a library of operations that a re FORTRAN subroutines. LINOLES are usually efficient routines for carrying out specific mathematical operations. Hovever, any BORTRAN subroutine can be joined to SPEAKEASY by an appropriate interface and become a LINKULE.

To obtain a listing of the present LINKULES, use the statements $T=$ L I BINDEX (KEPT)

TABULATE T

LIST MEMBER $X$ OF LIBRARY $Y$ FROM I TO $J$ is ists lines $I$ through $J$ OF $X$. $X$ is the name of a member of library to be listed. The words MEMBER, LIBRARY, PROM, and TO are keyvords. If TO is omitted, the listing begins at line $I$ and goes to the end of the member. If $P R O M$ is also omitted, the entire member is listed. LIBRARY Y specifies the library to be searched for the member. The default library is PRoClIB. If the nember is not specified, the library index is listed.

LIST specifies that the program is to be listed with the output. If not specified, the default for batch jobs is usually LIST.

In the interactive operation. NolIST is default.

girst in the edit mode lists the edit fille. The entire file is listed unless statement numbers follow the word LIST.

XLIST $N$ in the edit mode lists line $N$ of the edit file.

XLIST $N, M$ in the edit mode lists lines $N$ through $M$.

LISTHEAD(N,XX,YY) lists $N$ lines of nember $X X$ of library $Y Y$. $N$ specifies the maximum $n$ uber of lines of $X X$ to be listed. $X X$ is the name of the member to be listed.

$Y Y$ is the name of the library to be searched for member $X X$. If $Y Y$ is not specified, PRoclIB is default. 
LISTUEABBB ( $\left.X X_{g} I Y\right)$ lists neaber XX of library YY.

$X X$ is the name of the member to be listed.

$Y Y$ is the name of the library.

If $Y Y$ is owitted, the default is PROCLIB.

The entire nember is listed.

An alternative form is LIST MEMBER XX OP LIBRARY YY.

LOADDATA (A,MAE) loads a with Nalues from the data file NAME. $N$ is the number of elements of $A$ or the number of numbers in NAME, whichever is smaller.

LOADDATA is a restricted word.

LOC is a synongm for LOCS.

LOC ( $X$ ) gives the indices of the nonzero (true) elements of $x$. $X$ is a 1-dimenional object. LOC is a logical function and the argumert $x$ is of ten an array with a relational operator. The result is usually used as a structured index to produce a new array.

LOCMAX ( $x)$ specifies the location of the maximum element of $x$. $x$ must be a 1 -dimensional array:

LOCMIN ( $X$ ) specifies the location of the rinimum element of $x$. $x$ must be a 1-dimensional array.

LOCS(X) gives the indices of the nonzero (true) elements of $x$. $x$ is a 1-dimensional object. Locs is a logical function and the argument is often an array uith a relational operator. The result is usually used as a structured index to produce a new array.

LOC is a synonym for LOCS:

LOG $(x)$ returns the natural logaritha of $x$. LOG $(X)$ defines an object with the same structure as $x$ but with elements equal to the natural logarith of the corresponding elements of $x$.

LOG $(X)$ is not defined if any element in $x$ is 0 .

LOGGAMMA $(X)$ defines the natural logarithm of the gama function of $x$. LOGGAMMA $(X)$ defines an object with the same structure as $X$ but with elements that are the natural logarithm of the gamm function of the corresponding elements of $x$.

The elements $X i$ of $X$ must be real and satisfy the condition $0<X i<4 E 60$.

LOREBTRI $(X)$ returns the lover triangular part of $X$. The result is a 1 -dimensional object of the same family as $x$ with elements corresponding to the lower triangular elements and the diagonal elements of $x$.

$x$ aust be a 2-dinensional square object. 
- LT. is the relational operator "less than". The periods nust be on both sides of the aperator.

MARGINS enables the user to contol the margins of the output. MARGIN $(N, M)$ specifies the left and right limits of output. MARGINS(N,A) means that the output is restricted to columns starting at $N$ and ending with $M$.

MARGINS (M) is equivalent to MARGINS $(1, M)$.

MARGINS $(0, M)$ is equal to MARGINS ( $1, M)$. without control functions. MARGINS (O,M) eliminates all carriage control characters that are used to control the vertical spacing and to position output on the top of a ney page.

MaT is a synonym for MatrIX.

MAT (N, $M=)$ defines an $N-b_{Y}-M$ matrix.

If no additional arguments are given, the matrix has all elements set to zero.

MaT(N,M:I,J .....K) defines an $N-b y-M$ matrix and presets the elements. The elements are set row by row by use of the values $I, J, \ldots, K$ or the elements of $I, J, \ldots, K$ if they are structured objects. If a complex element is encountered, then a complex matrix is defined. If all the elements are not preset by the element list, the unspecified elements are set to zero.

MATRIX (N, $M:)$ defines an $N-b y-M$ matrix.

If no additional arguments are present, the matrix has all elements set to zero.

A shortened form is Mat.

MaTRIX (N,M:I,J,...,K) defines an $N-b y-n$ matrix with preset elements. The elements are set row by row by use of the values $I, J, \ldots, K$ or the elements of $I, J, \ldots . K$ if they are structured objects. If a complex element is encountered, then a complex atrix is defined. If all the elements are not specified by the element list, the unspecified elements are set to zero.

$\operatorname{Max}(X)$ specifies the value of the maximum element in $x$.

MaXof $(A, B, \ldots, C)$ returns the largest element of $A, B, \ldots, C$. The result is a scalar that is the maximum element in any of the objects in the calling argument list. The arguments must be real objects or numbers and can be of any class.

MaXOPCOL $(X)$ returns the largest eleaent in each column of $x$. $x$ is a 2-dimensional array or a matrix. The result is a 1-dimensional object of the same family as $X$ with elements that are the largest element of each colunn of $x$.

MAXOFBON(X) returns the largest element in each row of $X$. $x$ is a 2-dimensional array or a matrix. The result is a 1-dimensional object of the same fanily as $x$ ith elenents that are the largest elenent of each rou of $x$. 
MELD $(I, J, \ldots, K)$ gives an odoveter ordering of elements of $I, J, \ldots, K$. $T$ he arguaents $I, J, \ldots, K$ ast be 1 -dimensional objects. MELD alters the structure of $I, J, \ldots . K$ so that every elenent in each object of the argument $l i s t$ is associated with every element of every other object in the list.

After the statement MELD $(I, J, \ldots, K)$, any function of $I, J, \ldots, K$ can be vritten in a straightforward maner.

For a further explanation and examples, refer to the SPEAKEASY-3 manual or the linkule document.

MFaM (X) defines a member of the matrix/viector family. The result has the same structure as $X$. If $X$ is a 1-dimensional array or a vector, the result is a vector. If $X$ is a 2 -dimensional array or a matrix, the result is a matrix.

MFAM and VPAM are synonyms.

MIN $(X)$ specifies the value of the minimum element in $x$.

MINOF (A,B,...,C) returns the smallest element in $A, B, \ldots, C$. The result is a scalar that is the minimum element in any of the objects in the calling argument list. The arguments must be real objects or numbers and can be of any class.

MINOFCOL $(X)$ returns the smallest element in each column of $x$. $x$ is a 2-dimensional array or a matrix. The result is a 1-dimensional object of the same $f$ amily as $X$ with elements that are the smallest element of each column of $x$.

MINOFROH $(X)$ returns the smallest element in each row of $X$. $x$ is a 2-dimensional array or a matrix. The result is a 1-dimensional object of the same family as $x$ with elements that are the smallest element of each row of $x$.

XMOVE $N, M, K(I)$ moves statements $N$ through $M$ to $K, K+I, K+2 I, \ldots$ XMOVE $N, M, K$ moves statements $N$ through to $K, K+1, K+2, \ldots$ XMOVE $N$ moves statement $N$ to the last pasition. XHOVE $N, M$ moves statements $N$ through $M$ to the last positions. Hove is a function available in the EDIT mode.

MyDOCS is the library name for private documents. MYDOCS is the library name for private documents other than the private HELP documents that are attached to the SPEAKEASY procesSOr.

MYHELP is the library name for private HELP documents. GHELP is the library naie for private HELP docunents that are attached to the SPEARBAS processor. 
WYIIKS is the library name for private linkules. MYIINK is the library name for private linkules to be attached to the SPEAKEASY processor. It is of the same form as the systea library but contains personal routines. If MYIIAKS is an at tached library. it will be searched for a given member prior to the search of the LINKULE library.

Nanes prints the names of all curcently defined. SPEAKeasy objects. The comand NAMES is particularly useful. in the interactive mode since it provides the user with means of obtaining a listing of the names of variables that he has previousiy defined.

-NE. is the relational operator "not equal to". The periods must appear on both sides of the operator.

NEUMANN $(N U, X)$ returns cylindrical Bessel fins. of the second $k i n d$. NI and $X$ are real and nonnegative. There is a SPAREASY

restiction that $(X+N U)<400$.

For further details of accuracy and the method used for the calculation refer to the linkule document.

NEWGRAPH causes the next graph to be drawn on a new area of paper. NEWGRAPH may be used in conjunction with ADDGRAPH to completely aroid the use of the statement GRAPH.

NEHGRAPH is available only in version GRAPHEZ.

NEnPAGE causes the printer to start a new page. The output following the control statenent NEWPAGE is printed on the top of the next fage.

NEWPAGE is a restricted word.

Nocols $(x)$ defines a scalar which is the number of colums in $x$.

NOECHO suppresses the, echoing of input data.

This command causes just the results to be printed out. This statement may occur at any place in the program.

NOECHO is default for the interactive operation; ECHO is default for batch jobs.

NOELS $(X)$ defines a scalar equal to the number of elements in $x$. If $X$ is undefined, the result is zero.

A synonya is LENGTH.

NOLIST indicates that the program is not to be printed with the output. If not specified. NOIIST is default for the interactive

operation; IIST is usually default for batch jobs. 
HoRoots (P) gives the number of roots of the function $F$. The nuber of times the function $P$ chinges sign (goes through zero) is found.

A synonym is NOZEROS.

NOROWS $(X)$ defines a scalar equal to the number of rows in $X$.

- NOT. is the logical operator "not".

The periods must be on both sides of the operator.

NOZEROS (8) gives the number of zeros of the function $F$. The number of times the function $F$ changes sign (goes through zero) is found.

A synonym is NOROOTS.

NUMBERS refers to numerical data.

All numerical data are specified in decimal form. The lack of a decimal point implies that it belongs just to the right of the last digit.

NOMBEBS can be rea l, imaginary, or complex.

A terminal I implies that the previous number was imaginary. Examples of imaginary and complex numbers are: $21.61 ; 6.1+5.21$

Very large or very small numbers may be expressed by ending the number in $E$ followed by the power of 10 associated with that number. Por example, 1.052E+2 means 105.2. If a number is imaginary, the I comes before the E, eg.. 6. 2IE-3.

OBJECT (I,J,...K) generates an inplace name.

Each of the arguments I.J.....K can be either a literal quantity or a nonnegative number. Por literals, the expression itself

is used. Por numbers, the integer part of the number is used

as a literal. The various arguments are then joined together

to compose the name.

The expression OBJECT (I,J, ...R) may occur anyuhere in a SPEAREASY statement. It is particularly usefull combined with the WOrd HENCEPORTH.

OMITCLASS suppresses the printing of the class. If OMITCLASS is not specified, the class of the resulting structured objects is printed out when they are printed. The statement OMITCLASS suppresses the printing of the class.

ONRRROR $(X, Y)$ specifies the action to be taken af ter an error. $X$ ma $Y$ be DUMP or NODUMP.

The options DUMP. or NODUMP specify whether all defined data is to be printed or not.

$Y$ may be MANUAL Or CONTINUE.

MANUAL means that computation is continued in the manual mode. CONTINOE means that the error does not affect the path of execution.

The default options are DUMP, BAnual. 
$-O R$. is the logical operator "or".

The periods nust be on both sides of the aperator.

ORDERED $(X)$ gives the elements of $x$ in increasing order. ORDERED(X) defines an object with the same structure as $X$ but whose elements are the elements of $x$ arranged in order of increasing magnitude.

A SYRONYW is RANRED.

ORDERER $(x)$ gives $t$ he indices of the ordered elements of $x$. ORDERER ( $X$ ) gives the indices of the elemenits of $X$ arranged in order of increasing magnitude. $x$ must be a 1-dimensional object.

A syoony is RANKER.

PLOTSYMB (N,M) specifies the symbols used and frequency of points. N is an integer which determines the frequency ith which each symbol is plotted. (1 means every point, 2 means every other point, 3 means every third, etc.) A negative value for $N$ indicates that only the symol should appear. A positive value means that a line should join successive points.

$M$ is an integer 0 through 12 that designates one of 13 different symols to be used in plotting data. The integers and their corresponding symbols are:

0 is a square.

1 is a circle.

2 is a triangle.

3 is a plus $(+)$.

4 is an $X$.

5 is a diamond.

6 is an arrow pointing upward.

7 is an $X$ with a bar on top.

8 is a $Z$.

9 is a $Y$.

10 is an $X$ with a circle.

11 is an asterisk (*).

12 is an $X$ with a bar on top and bottom.

pLotsya is used for graphical output.

PRINT (A,B, C, ...Z) specifies that $A, B, C, \ldots \ldots z$ are to be printed. The user day control the form of output by using the control words MARGINS, SIGNIPICANCE, AUTOTAB, TABOLATE, and COLHIDTH.

PRINT is a restricted word.

PRINTCLASS prints the class of structured objects. PRINTCLASS causes the class of structured objects to be printed when the structured objects are printed. PRINTCLASS is default. To turn it off, use OHITCLASS.

PROD $(X)$ defines the product of the elements of $X$. The result is a scalar. 
PRODCols $(X)$ nultiplies the elements in each colum of $X$. PRODCOLS (X) defines a 2-dimensional object that is a row and is a member of the family of $x$. The ele ment in each column is the product of the elements. in the corresponding column of $X$.

PRODBORS $(X)$ multiplies the elements in each row of $x$. PRODRONS $(X)$ defines a 2-dimensional object that is a column and is a member of the family of $x$. The element in each row is the product of the elements in the corresponding row of $\mathrm{X}$.

PROGRAM NAME gives a name to a program.

A program is a SPEAKEASY procedure. Maming a program enables the user to refer to that program by mame to stora it, to use it within another program, or to execute it.

a program is a defined object. If its name is identical to a previously defined object, its definition vill replace that object.

PROGRAM is a restricted vord.

PUNCH $(F=X)$ punches the object $X$ on cards in the format $P$. The PONCA statement gives the user a means of transmitting infornation to other non-SPEAREASY prograns.

The format $P$ is specified by a statement of the form $P=$ (FORMAT) where PORHAT is the standard 360-FORTRAN IV format excluding fixed-point form (integers): The F, D, and $E$ formats are equally acceptable.

$x$ is the array to be punched.

QUIT terminates execution of a SPEAREASY, program. When all input is processed in normal batoh operation, execution autonatically terminates. This word is therefore not normally used for such cases.

BANDOM $(X)$ generates random numbers. The resuit has the same class and structure as $X$ but its elements are random numers between 0.0 and 1.0 .

BANDOG yill produce the same numbers on successive calls unless the first use of it is preceded by the statement LOAD (RANDOM).

RANKED $(X)$ gives the elements of $X$ in increasing order. RANRED (X) defines an object with the same structure as $X$ but whose elements are the elements of $X$ in increasing order.

A Synonym is ORDERED.

RANKER (X) gives the indices of the ordered elements of $X$. RANKER (X) gives the indices of the elements of $X$ arranged in order of increasiag agnitude. I nust be a 1-dimensional ob ject.

A synonym is ORDERBR. 
BBAD(F:X) reads data fron cards panthed in format $F$ and puts thea into $X_{\text {. }}$. The READ statenent enables the user to read information that has been punched in some specialized forat.

The format $P$ is specified by a statement of the form $P='$ (FORHAT) ' where FORAAT is the standard 360-PORTRAN IV fornat excluding fixed-point for (integers).

$x$ is the array (structured object) into which the data are put. It wust exist and its size must be specified. The number of cards read is the number needed to fill the array.

READ is not available in all versions of SPEARBASY.

REAL is a synonym for REALPABT.

REAL $(X)$ returns the real part of $X$. REAL $(X)$ defines an object vith the same structure as $X$ but the elements of the result are the real part of the corresponding elements of $X$.

REALPART $(X)$ returns the real part of $x$. REALPART $(X)$ defines an object with the same structure as $x$ but the elements of the result are the real part of the corresponding elements of $x$.

a synonym is REAL.

RBAL $4(X)$ is a function that returns a real 4 number. $x$ may be real 8 , integer4, or integer2. The result is real4.

REAL $8(X)$ is a function that returns a real 8 number. $X$ may be real4, integer 4 , or integer 2 . The result is real8.

RECLASS (A:B,C,.., Z) alters the structure of $B, C, \ldots, Z$ to agree with $A$. B, C,.., Z are previously defined objects with the same number of elements as $A$.

RETURN returns execution to the program calling the stored program. The statement executed after the statement RETURN is the one following the EXECOTE statement that invoked the stored program. A BETURN statement is alvays implied before the END card of any program.

RETURN is restricted to the program mode. It is ignored if it is used in the manual mode.

RETURN is a restricted word.

BOOTS $(F: X)$ finds the roots of the function $F$. The result is a $1-d i m e n s i o n a l$ array. $X$ is a 1-dimensional array of the same length as $F$. Roots defines a new object whose elements correspond to the zeros of the function P. The method of trapezoidal interpolation is used.

a synonym is zeros. 
BOHARRAY (N:) defines a 2-din. N-conponent array that is a row. If no further arguments are given, all $N$-conponents are set equal to zero.

ROWARRAY $(: I, J, \ldots, R)$ defines a 2 -din. rov array with preset els. The components a re preset by the element 1 ist $I, J, \ldots, K$. If any argument of the element list is structured, then the elements of that structured object are used. If a complex elenent is encountered, then a complex 2-dimensional row array is defined.

BOWARRAY (N:I,J,....K) defines a 2-dim. N-conponent rov arrayThe components are preset by the element list $I, J, \ldots . K$. If any argument of the element list is structured, then the elements of that structured object are used. If a complex element is encountered, then a complex 2-aimensional rov array is defined. If all $\mathrm{N}$-components are not preset by the element list, the unspecified components are set equal to zero.

Rownat (N:) defines a 1-by-N matrix which is a row. If no further arguments are given, all $N$ elements are set equal to zero.

ROWHAT (:I,J,....K) defines a roy matrix and specifies the els. The element list $I, J, \ldots . . K$ specifies the elements of the matrix. If an argument of the list is structured, the elements of that structured object are used. If one of the elements is complex. the watrix is complex.

BOWMAT (N:I,J,....K) defines a 1-by-N row matrix and specifies the els. The elements are preset by the element list $I, J, \ldots, K$. If an argument of the element list is structured, the elements of that structured object are used. If an element is complex, the matrix is complex. If all $\mathrm{N}$ elements are not specified by the element list, the unspecified elements are set equal to zero.

ROWMAX (X) specifies the cow containing the maximum element of $x$.

ROWMIN $(X)$ specifies the row containing the minimum element of $X$.

TRON is equivalent to XCOMPILE followed by the command EXECUTE. TBUN used in the EDIT mode returns the processor from the EDIT mode to the Mandal mode, compiles the editied program, and then executes it.

SELECT (A,B,....C:I) truncates or expands $A, B, \ldots, C$ using the index $I$. $A, B, \ldots . C$ are 1-dimensional objects. I is a structured index that acts as a control array. A, B..... C are truncated or expanded so their final components are those indexed by the elements of the control array $I$.

For an example, refer to the SPEAKEASY-3 wanual. 
SETGAUSS (N, $X, H, X I O, X H I)$ returns Ganss-Legendre coords. and veights. SETGAUSS ( $N, X, W, X L O, X B I$ ) defines the ${ }^{4}$ point Gauss-legendre quadrature coordinates and weights with the coordinates in the interval XLO to XHI. The arguments XLO and XHI may be omitted. If XLO and XHI are o 1 itted, the interval is -1 to +1 .

$N$ is the number of points required. Inst satisfy the condition $0<N<51$.

$X$ is returned as a 1 -dimensional array uhose elements are the coordinates.

D is returned as a 1 -dimensional array whose elements are the veights.

For further information about the method of calculation and the accuracy, refer to the LINKULE document.

SETINFINITY (VAL) specifies an upper linit to the numbers printed. Any number whose absolute value is greater $t$ han val is printed as INP for infinity. The default value is 1. $E+30$.

SBTJACOBI (N,X,H,A, B,TX,TH) defines Gauss-Jacobi coords, and weights. SBTJACOBI (N,X,H,A,B, IX,TR) defines the Gauss-Jacobi quadrature coordinates and weights for the interval -1 to +1 and gives the user an idea of the precision with which the coordinates and weights are calculated. The coordinates are the roots of the Jacobi polynomial of degree $N$ and order $(A, B)$. A, B, TX, and $T$, are optional arguments and may be omitted:- If $A$ and $B$ are onitted, the order of the polynomial is 0 .

$N$ is the number of points required. N wust be an integer satisfying the condition $0<N<51$.

$X$ is returned as a $1-d i m e n s i o n a l$ array whose elements are the roots of the Jacobi polynomial of degree and order $(A, B)$.

is returned as a 1 -dimensional array wose elements are

the weights of the integration proceedure.

$A$ and $B$ are real numbers specifying the order of the polynomial. A must satisfy the condition $A>-1$. B nust satisfy the condition B>1. The default value for $A$ and $B$ is 0 .

$T X$ is the theoretical sum of the coordinates.

$T W$ is the theoretical sum of the weights.

Por furtber information about the method of calculation and the accuracy. refer to the LINKOLE document.

SETLAGUEBBE ( $N, X, H, A)$ returns Gauss-Laguerre coords. and reights. SETLAGUERRE (N,X,H,A) defines the Gauss-Laguerre quadrature integration points $X$ and reights appropriate for evaluating an integral from 0 to infinity. The integration points are the roots of the Laguerre polynonial of degree $N$ and order $A$. $A$ may be ouitted. If $A$ is omitted, the polynomial calculated is order 0 .

$N$ is the number of points required. N nust be an integer and satisfy the condition $1<N<33$.

$X$ is returned as a 1-dimensional array whose elements are the roots of the Laguerre polynomial of order $A$ and degree $N$.

$W$ is returned as a 1-dimensional array vith the veights of the integration proceedure.

A is the order of the polynomial. The lefault is 0 . A nust be a real nuaber greater than -1. A value of $A$ of the order 20 could cause overflous in the determination of the veights for large values of $\mathrm{n}$. 
SETLEGENDRE $(N, X, W)$ returas Gauss-Legendre coord. and ueights. SBTLEGEIDRE $(N, X, X)$ returns the coordinates $X$ and veights $N$ for the Nth order Gauss-Legendre guadrature formula.

$\checkmark$ is the number of points required. N aust satisfy one of the conditions $0<N<51, N=64$, or $N=96$.

$X$ is returned as a $1-d i m e n s i o n a l$ artay whose elements are the coordinates.

W is returned as a 1-dimensional arsay whose elements are the veights.

SETLEGENDRE is equivalent to SETGAOSS for $0<N<51$. The only difference is that SETLEGENDRE includes $N=64$ and $\mathrm{N}=96$ while SETGAUSS does not.

SETLIB $(X X, Y Y)$ changes the name of library XX to YY.

SETNULL (VAL) specifies a lower limit to numbers printed. An number wose absclute value is less than VAL is to be printed as 0 . The default value is 1:.E-30.

SETPLOT $(X, Y, Z)$ specifies BOX, NO BOX;: SCALBS, NOSCALES; LINES, POIATS. $X$ is either BOX or NOBOX. Box means to draw a frame around the graph. NOBOX specifies no frame.

$I$ is either SCALES or NOSCALES.

SCALES specifies that values at inch intervals are to be indicated. NOSCALES causes the indication of scales to be omitted.

$z$ is either LINES or POINTS.

LINES specifies that points are to be joined by lines leaving

the points unarked. PoI N s secifies that the points are

to be marked vith crosses and not joined by lines.

The default options are BOX, SCALES, and LINBS.

SETPLOT is available only in version GRAPHEZ.

SIGN $(X)$ specifies whetber $X$ is positive or negative. SIGN(X) defines. an object with the sane structure as $X$ but with elements of the result equal to +1 for elements of $x>0$, equal to -1 for elements of $x<0$, and equal to 0 for elements of $x$ equal to 0 .

All the elements of $x$ must be real.

SIGNIFICARCE(N) gives the number of significant figures to be printed. is is the number of significant figures desired. The default is 5 significant figures.

SIHEQ $(A, B)$ solves a set of simultaneous linear equations. $A$ is an $N-b y-N$ matrix. B is an $N-b Y-n$ matrix of constants. The result, $X$ has the same structure as $B$ and is the matrix that satisfies the $s$ inultaneous lineas equation $A * X=B$. 
SIN(X) returns the sine of $x$.

SIN( $x)$ defines an object ith the same structure as $x$ but

the elements of the result are the sine of the

corresponding elements of $x$.

Each element $X i$ of $X$ must satisfy the condition

$|X i|<1.815$.

SIN $(x)$ defines the hyperbolic sine of $x$.

SINH $(X)$ defines an object with the sane structure as $X$ but with elements equal to the hyperbolic sine of the corresponding elements of $x$.

The elements $X i$ of $x$ must be real and are restricted by the inequality $|x i|<170$.

SIZE=N, $X$ specifies space for data and wust be the first card. $S I Z E=N, Z$ specifies the amount of data space needed and its location.

$N$ is the number of kilobytes of storage to be set aside for SPEAKEASY data. one kilobyte is approximately 120 user-defined numbers.

$x$ may be MAIN or LCS. LCS means the data storage area may be placed in LCS. MAIN is default.

The card specifying the size is the first one in the SPEAREASY deck.

SHAT is a synonym for SYGMAT.

SMAT (N:I,J,...K) defines a symetric $N-b y-N$ matrix.

The element list is used to fill the lower triangular part

by rows. The portion above the diagonal is then filled by

making the matrix symetric. If any argunent in the list

defining the elements is structured. the elements of that

structured object are used.

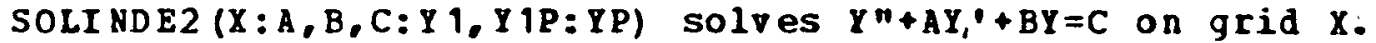

$Y=\operatorname{SOLINDE2}(X, A, B, C: Y 1, Y$ 1P:YP) is the tYpical usage of the function

SOLINDE2. The colons may be replaced by comas. $X, A, B, C, Y P$,

and $Y$ are all 1 -dimensional arrays.

$X$ is an equally-spaced grid of independent variables.

It may be an increasing or decreasing grid.

$A, B$, and $C$ are coefficient functions evaluated on the grid $X$.

$Y 1$ is the starting value of $Y$ at $X(1)$.

$Y Y^{\prime}$ is the starting value of $Y$ ' at $X(1)$.

$Y$ is the solution on grid $X$ and is returned as a functional value.

$Y P$ is the derivative of the solution on grid $x$. YP is an optional

name and need not be the name of a defined object. If $Y P$ is

onitted, the calculation proceeds unaltered but $Y^{\prime}$ is not saved.

Tenporary restrictions are:

$A$ and $B$ wust be named scalars with the value zero. only homogeneous equations ithout first derivatives can be solved. An example is $Z=0 ; \quad Y=S O L I N D B 2(X: Z, B, Z: Y 1, Y 1 P: Y P)$.

$X$ must have an odd number of points. Ho internal shecks are wade.

The 4 th order Runge-Rutta method is used.

SPACE(B) skips lines.

SPACE is a restricted word. 
SPHBES $(L, X)$ returas the spherical Bessel fa. of the first kind. The result is $j$ sub $I$ of $X$ (little $j$ ).: L wust be a nonnegative integer. onderflow vill occur for excessively large $L$. $X$ ust be real and nonnegative. There is a SPBAREASI restriction that $(X+I)<400$.

Por information on the aethod of calculation, refer to the LINROLE document.

SPHBESN $(L, X)$ returns the spherical Bessel fn. of the second kind. The result is $n$ sub $L$ of $X$ (little $n$ ). L must be a nonnegative integer. overflow vill occur for excessively large $L$. $x$ must be real and nonnegative. There is a spEAKEASY restriction that $(L+X)<400$.

Por information on the method of calculation, refer to the LIRKOLE document.

SQRT (X) defines the square root of $X$. SQRT $(X)$ defines an object with the sane structure as $X$ but with elements equal to the square root of the corresponding elements of $X$.

If the domain of the calculation is complex, complex roots can be obtained. othervise, the square root of a negative number leads to an error message.

SuM $(x)$ sums the elements of $x$. SuM(X) defines a scalar object equal to the sum of the elements of $x$.

sumcols $(X)$ sums the elements in each col uin of $x$. surcols $(X)$ defines a 2-diaensional object that is a row and is a member of the family of $X$. The element in each column is the sum of the elements in the corresponding column of $x$.

SUMROMS $(X)$ sums the elements in each row of $x$. SUAROHS $(X)$ defines a 2-dimensional object that is a column and is a member of the family of $x$. The element in each row is the sur of all the elements in the corresponding row of $x$.

SUMSQ( $x$ ) sums the squares of the elements of $x$. SUMSQ $(X)$ defines a scalar that is the sum of the squares of all the elements of $x$.

SOMSQCOLS $(X)$ sums the squares of the elements in each column. SomSQCOLS $(X)$ defines a 2-dimensional objeot that is a ror and is a member of the same family as $X$. The element in each colum is the sum of the squares of elements in the corresponding colung of $x$. 
solsogons (X) suns the squares of the elenents in each row. SURSQROnS $(x)$ defines a 2-dimensional objeat that is a colum and is a member of the fanily of $x$. The element in. each row is the sum of the squares of the elements in the corresponding row of $x$.

SYMBOLS designate nathematical operations or special cards. The symols and a brief description of their meanings are:

+ is the addition operator.

- is the subtraction operator.

* is the nultiplication operator.

For matrices, * implies matrix multiplication.

For vectors, * implies take the inner (dot) product.

For arrays, * is element-by-element multiplication.

If $A$ and $B$ are 1-dim. arrays, $C=A * B$ is $C I=A i * B i$, were $i$ implies

the ith element.

/ is the division operator.

For matrices, A/B implies matrix nultiplication of the matrix times the inverse of matrix $B$.

For vectors, / is not defined.

Por arrays, division is defined element-by-element.

* raises to a power.

* is not defined for matrices.

For vectors, * means to take the outer (cross) product.

For arrays, the operation is done element-by-element.

= means replace the object on the left by the expression on

the right.

: separates the statement label from the statement or

separates arguments in some words.

: separates successive statements on a single card.

\& designates a continuation of the previous card.

$\$$ sets off a comment. The coment is written between tro $\$$. There is an implied \$ at the end of a card that has a single $\$$ on it.

SYMMAT (N:I,J,...K) defines a symetric N-by-N watrix. The elenent list is used to fill the lover triangular part by rows. The portion above the diagonal is then filled by making the matrix symetric. If any argument in the element list is structured, the elements of that structured object are ased.

a shortened form is SMAT.

TABOLATE (A,B,...,C) prints 1-dim. objects $A, B, \ldots .$, in tabular form. Each column is labeled by the name of the corresponding object.

The user may control the tabulation by the specifications MARGINS, COLKIDTH, and SIGNIPICANCE. All tables may be made uniform by using AUTOTAB.

Only 1-dimensional objects ay be tabulated.

TAN ( $x)$ returns the tangent of $x$.

ran (X) defines an object with the samer structure as $x$ but

the elements of the result are the tangents of the

corresponding elevents of $x$.

Each element $X i$ of $X$ nust satisfy the condition $|X i|<1 . E 15$. 
TInB gives the time fros which one starts.

TInE gives the total tine elapsed since the start of

the conputer run.

TInE(0) gives the elapsed time since the start of a computer run. This for can be used in SPEAREASY statements.

TOTALINT $(P: X)$ defines the definite integral of $F$ over the array $X$. $X$ is a 1 -digensional array. $(X 1, X 2, X 3, \ldots, X N)$. The result is a scalar equal to the definite integral from $X 1$ to $X N$. The integration is done by the trapezoidal rule.

A shortened form is TorInT.

TOTINT is a synony for TOTALI MT.

TOTINT $(F=X)$ defines the definite integral of $F$ over the array $X$. $x$ is a 1 -dimensional array $(X 1, \times 2, \times 3, \ldots, X M)$. The result is a scalar equal to the definite integral from $x 1$ to $x H$. The integration is done by the trapezoidal rule.

TRACE $(X)$ gives the trace of the natrix $x$. The trace is the sum of the diagonal elements.

TRANSP is a synony for TRANSPOSE.

TRANSP $(X)$ defines the transpose of $X$. TRANSP $(X)$ defines an object that is of the same class as $X$ and is the transpose of the object $x$.

TRANSPOSE $(X)$ defines the transpose of $x$. TRANSPOSE $(X)$ defines an object that is of the same class as $x$ and is the transpose of the object $x$.

A shortened form is TRANSP.

UMAT is a synonym for UNITHAT.

OMAT (N) defines an N-by-N unit matrix. A11 elements are zero except the elements along the diagonal, which have the value 1.

ONITHAT(N) defines an $N-b y-N$ unit matrix. All elements are zero except the elements along the diagonal, which have the value 1.

A shortened form is UMAT.

UPPERTRI (X) returns the upper triangular part of $X$. $x$ ast be a square 2 -dimensional object. The result is a 1-dimensional object that is of the same family as $X$ and wose elements are the upper triangular elements including those along the diagonal of $x$.

USE HEMBER X OP LIBRARY $Y$ causes the deak $X$ to be used as input. If the library reference is onitted, PROCLIB is assumed. If the library uYPRoCs is attached, it will be searched for the member $X$ before the library proclib is searched.

This provides the user with a simple means of supplying commonly used constants or SPEAKeASY programs to the processor.

USE is a restricted word. 
USEMEMBER $(X X, Y Y)$ causes input of the neaber $X X$ of library $Y Y$. $X X$ is the name of the member desired.

$Y Y$ is the name of the library to be searched for member $X X$.

If the library reference is onit ted. PROCLIB is assumed. If the library nYPROCs is attached, it will be searched for wember $X X$ before PROCLIB is searched.

This provides the user with a means of supplying commonly used constants or SPEAKEASY programs to the processor.

An alternative for is USE MEMBER XX OP LIBRARY YY.

VABIABLE(I,J) defines a 1-dia. array of 101 points from $I$ to J. The increment is chosen by the conputer sa that the points are equally spaced. Both points $I$ and $J$ are included.

A synonym is GRID (I, J).

VARIABL $B(I, J, K)$ defines the 1 -dim. array $I, I+R, I+2 K, \ldots, J$. The last element in the array is $J$. If the increment $K$ is not such that $I+N R=J$, then a new increment $R^{\prime}$ is chosen by the computer so that the last element is $J$ and the elements are equally spaced.

VARIABLE $(I, J, K)$ is equivalent to $G R I D(I, J, K)$ only when $K$ is such that $I+N K=J$.

VEC is a synonym for VECTOR.

VEC (N:) defines a vector with $N$ conponents. If there are no additional arguments, the values of the components are set equal to zero.

$V E C(: I, J, \ldots, K)$ defines a vector and specifies the components. The components a re preset by the element $l$ ist $I, J, \ldots . . K$. If any argument of the element list is structured, then the elements of that structured object are used. If a complex element is encountered, then a conplex vector is defined.

VEC (N: I, J,...,K) defines an $\mathbb{N}$-component vector with preset els. The components are specified by the element list $I, J, \ldots . K$. If any argument of the element list is structured, then the elenents of that structured object are used. If a complex element is encountered, then complex vector is defined. If all $\mathrm{N}$ components are not specified, the unspecified components are set equal to zero. 
VECTOR (N:) defines an $\mathrm{N}$-component rector. If no further arguments are given, all the conponents are set equal to zero.

a vector is a 1-dimensional member of the matrix/vector fanily.

A shortened form is VEC.

VECTOR $(: I, J, \ldots ., R)$ defines a vector by presetting the components. The components are specified by the element list $I, J, \ldots . ., K$. If any argument in the element list is structured, then the elements of that structured object are used. If a complex element is encountered, a complex vector is defined.

VECTOR (N:I,J.....R) defines an $N$-component vector vith preset els. The components are preset by the elenent list $I, J, \ldots . ., K$. If any argument in the element list is structured, then the elements of that structured object are used. If a complex element is encountered, a complex vector is defined. If all $N$ components are not preset by the element list. the unspecified components are set to zero.

VERSIONS refers to the various versions of the SPEAKEASY processor. These versions differ in their space requirements and in their efficiency of operation. The present versions are:

STANDARD

BABY

GRAPHEZ

CONSOLE

TSO

For further detail about these versions, refer to the SPEAREASY-3 manual.

VFAM $(X)$ defines a member of the matrix/vector family. The resultant bas the same structure as $x$. If $x$ is a 1-dimensional array or a vector, the result is a vector. If $x$ is a 2-dimensional array or a matrix. the result is a inatriz.

VLABEL='ANY MESSAGE' labels the vertical scale. The desired label is enclosed between apostrophes. VLABEL is available only in version GRAPHEZ.

VOCABULARY generates a list of all currently defined words. The list is the actual list used by the processor in deciphering the user's SPEAKEASY program. The printout in response to the vord POCABULARY will change whenever a ney word is added to the language.

VSCALE= (BOTTOM,TOP) specifies the vertical limits of a graph. BOTTOM refers to the lover limit and rop the upper linit of the vertical scale. Default values of the limits on the vertical scale are 0 and 8 . The scale is labeled at inch warks.

VSCALB is used for rersions with graphical output. 
VSIZE=Y specifies the vertical size of the graph. $I$ is in inches. The default is 8 inches. $Y$ cannot exceed 10 .

VSIZE is used for versions with graphical output.

DHERE is a synonym for DREREVER.

WHERE $(X)$ is a conditional statement for array operations.

$X$ may be a structured object. The associated statement $Y$ must be an equation and the class of the result wust be the same as that of the test expression $X:$ : The statenent $Y$ is executed only if the test expression $X$ is true (nonzero).

WERE is a retricted vord.

WHBREVER $(X)$ Y is a conditional statement for array operations. $X$ may be a structured object. The associated statenent $Y$ must be an equation and the class of the result nust be the same as that of the test expression $X$. The statenent $I$ is executed only if the test expression $x$ is true (nonzero).

WHEREVER is a restricted word.

A shortened form is RHERE.

HHOL $E(X)$ returns the integer part of $X$.

DHOLE (X) defines an object with the same structure as $X$ but the elements of the result are the integer part of the corresponding ele ments of $x$.

A synonym is INTPART.

MRITE(F: $X)$ prints the defined object $X$ in the format $P$. The RRIT statement enables the user to print information in a specific format.

The format $P$ is specified by a statement of the form $F=$ ' (FORMAT) where PORAAT is the standard 360-FORTRAN IV format excluding fixed-point form (integers).

$X$ is the defined object to be printed in the specific format. URITE is not available in all versions of SPEAKEASY.

ZEROS(F:X) finds the zeros of the function $F$. The result is a 1 -dimensional array. $X$ is a 1-dimensional array of the same length as F. ZEROS defines a new object whose elements correspond to the zeros of the function

P. The method of trapezoidal interpolation is used.

A synonym is ROOTS. 


\section{APPENDIX \\ HELP documents are written as follows. Each} word in the HELP documents is written in a specific format for retrieval of various levels of information. The first line of each document may be obtained by itself. Consequently, it is a one-line definition of the word. The information in each document is structured in levels so that each paragraph goes into greater detail. Eventually, the printing of a document will be halted after each paragraph so that the user will have the option of stopping or requesting further information. If further information is requested, the next paragraph will be printed.

To facilitate obtaining output on various devices, the length of each line is limited to 72 characters. For uniformity, a new paragraph is indented two spaces. Thus a line that begins a new paragraph is limited to 70 characters.

The first line of each document consists of (1) the word being defined (in capital letters), (2) the arguments of the word (in capital letters), and (3) a concise definition of the word. Since the first line is the beginning of a paragraph, it is indented two spaces so 70 characters are left for the word, argument list, and definition. Ideally this should be a complete sentence. Sometimes abbreviations must be used in order to convey the meaning of the word in so'little space.

In addition to the restriction that the rest of the paragraph and the following paragraphs have lines of 72 characters or less, there is the convention that the word being defined and any of the arguments appear in capital letters throughout the document. If a word has more than one calling sequence, it is listed with each calling sequence as a new paragraph. The first line of this new paragraph begins with the word being defined and follows the same format as the first line of the document. 
The last line of the document should have the initials of the contributor in columns 68-72. The contributor as sumes responsibility for the validity of operation of the word described.

The documents created in this way are placed into a staging data set for validation before introducing them into the system library. However, the newly defined documents are available to. SPEAKEASY users at Argonne.

HELP documents are written in upper and lower case. Consequently, words may be put in only on devices that have both upper and lower case, e.g., on the IBM-2741 terminal. The command TMODE LOWER gives lower case letters in the RESCUE EDIT mode.

The definition of each word must be preceded by a card of the form

\section{/TO NEWWORD}

The / is in column 1. NEWWORD is the first 8 characters of the word being put into the HELP-document library. This card is in capital letters.

An example follows. 
//JFHELP JOB $(F 88888,1,1,1), C L A S S=C, R E G I O N=85 K$

$1 /$ EXEC SOS

$/ / L I B O U T$ DD DSN=SPEAK. NEW. HELP, DISP=OLD

/TO MATRIX

MATRIX(N,M:) defines an $N-b y-M$ matrix.

If no additional arguments are present, the matrix has all

elements set to zero.

A shortened form is MAT.

MATRIX $(N, M: I, J, \ldots, K)$ defines an $N-b y-M$ matrix with preset elements.

The elements are set row by row by use of the values $1, J, \ldots, k$ or

the elements of $1, \mathrm{~J}, \ldots, \mathrm{K}$ if they are structured objects. If

a complex element is encountered, then a complex matrix is

defined. If all the elements are not specified by the element

list, the unspecified elements are set to zero.

/TO SUMSQCOL

SUMSQCOLS $(x)$ sums the squares of the elements in each column. $\operatorname{SUMSQCOLS}(x)$ defines a 2-dimensional object that is a row and is a member of the same family as $x$. The element in each column is the sum of the squares of elements in the corresponding columns of $x$. 